\title{
The Hanle effect in a random magnetic field
}

\section{Dependence of the polarization on statistical properties of the magnetic field}

\author{
H. Frisch ${ }^{1}$, L. S. Anusha ${ }^{2,1}$, M. Sampoorna ${ }^{2}$, and K. N. Nagendra ${ }^{2}$ \\ 1 Université de Nice, Observatoire de la Côte d'Azur, CNRS, Laboratoire Cassiopée, BP 4229, 06304 Nice Cedex 4, France \\ e-mail: frisch@oca.eu \\ 2 Indian Institute of Astrophysics, Koramangala, Bangalore 560 034, India
}

Received 21 January 2009 / Accepted 18 March 2009

\begin{abstract}
Context. The Hanle effect is used to determine weak turbulent magnetic fields in the solar atmosphere, usually assuming that the angular distribution is isotropic, the magnetic field strength constant, and that micro-turbulence holds, i.e. that the magnetic field correlation length is much less than a photon mean free path.

Aims. To examine the sensitivity of turbulent magnetic field measurements to these assumptions, we study the dependence of Hanle effect on the magnetic field correlation length, its angular, and strength distributions.

Methods. We introduce a fairly general random magnetic field model characterized by a correlation length and a magnetic field vector distribution. Micro-turbulence is recovered when the correlation length goes to zero and macro-turbulence when it goes to infinity. Radiative transfer equations are established for the calculation of the mean Stokes parameters and they are solved numerically by a polarized approximate lambda iteration method.

Results. We show that optically thin spectral lines and optically very thick ones are insensitive to the correlation length of the magnetic field, while spectral lines with intermediate optical depths (around 10-100) show some sensitivity to this parameter. The result is interpreted in terms of the mean number of scattering events needed to create the surface polarization. It is shown that the single-scattering approximation holds good for thin and thick lines but may fail for lines with intermediate thickness. The dependence of the polarization on the magnetic field vector probability density function (PDF) is examined in the micro-turbulent limit. A few PDFs with different angular and strength distributions, but equal mean value of the magnetic field, are considered. It is found that the polarization is in general quite sensitive to the shape of the magnetic field strength PDF and somewhat to the angular distribution. Conclusions. The mean field derived from Hanle effect analysis of polarimetric data strongly depends on the choice of the field strength distribution used in the analysis. It is shown that micro-turbulence is in general a safe approximation.
\end{abstract}

Key words. line: formation - polarization - magnetic fields - radiative transfer

\section{Introduction}

As pointed out by Stenflo (1982, see also, 1994, 2009), the Hanle effect provides a powerful diagnostic for detecting the presence of a weak turbulent magnetic field. The physical origin of this field and symmetry properties of the observed linear polarization suggest that the field scale of variation is small compared to the mean free path of photons and hence that "microturbulence" could be assumed. This allows one to replace all the physical parameters depending on the magnetic field by their average over the magnetic field vector PDF (probability density function). All the determinations of solar turbulent magnetic fields have been carried out so far with this approximation (Faurobert-Scholl 1993, 1996; Faurobert 2001; Trujillo Bueno et al. 2004; Bommier et al. 2005; Faurobert et al. 2009). In addition, it is usually assumed that the magnetic field PDF is isotropically distributed and that its strength has a single value. The Hanle problem reduces then to a resonance polarization problem with a modified polarization parameter that is in general smaller (Stenflo 1982, 1994).

In a preceding paper (Frisch 2006, henceforth referred to as HF06), a model magnetic field has been introduced allowing one to examine the possible effects of a finite magnetic field correlation length (comparable to a typical photon mean free path). Equations have been established for calculating the mean Stokes parameters, but no numerical results were given. In the present paper, the equations given in HF06 are rewritten in a form easily amenable to a numerical solution. An iterative method of solution of the ALI type (approximate lambda iteration) is used to calculate the mean Stokes parameters. We examine their dependence on the correlation length of the magnetic field and analyze the results in terms of the mean number of scattering events contributing to the formation of the surface polarization. We also investigate the sensitivity of the mean Stokes parameters to the shape of the magnetic field PDF, the objective being to see whether the Hanle effect can provide some clue to the behavior of this quantity.

In Sect. 2, we describe the magnetic field, the atomic and atmospheric models (they are the same as in HF06). We establish the transfer equations for the calculation of the mean Stokes parameters in Sect. 3. In Sect. 4 we describe an ALI type numerical method of solution. In Sect. 5 we describe different types of PDFs used in our investigation. The finite correlation effects are presented in Sect. 6 and analyzed in Sect. 7. Finally, in Sect. 8, we calculate the mean polarization for various types of magnetic field strength PDFs, in the framework of micro-turbulence. Some 
technical details about transfer equations and calculations of the mean Stokes parameters are presented in Appendices A and B.

\section{Assumptions}

We consider a two-level atom with unpolarized ground-level and assume complete frequency redistribution. The $4 \times 4$ redistribution matrix takes the form

$R\left(x, \boldsymbol{n}, x^{\prime}, \boldsymbol{n}^{\prime} ; \boldsymbol{B}\right)=\varphi(x) \varphi\left(x^{\prime}\right) P^{\mathrm{P}}\left(\boldsymbol{n}, \boldsymbol{n}^{\prime} ; \boldsymbol{B}\right)$,

where $x^{\prime}$ and $x$ are the frequencies of incident and scattered beams measured in Doppler width units from line center and $\boldsymbol{n}^{\prime}$, $\boldsymbol{n}$ their directions. The function $\varphi(x)$ is the line absorption profile normalized to unity. The elements of the polarization matrix can be written in the form

$$
\begin{aligned}
& P_{i j}^{\mathrm{P}}\left(\boldsymbol{n}, \boldsymbol{n}^{\prime} ; \boldsymbol{B}\right)= \\
& \quad \sum_{K Q} \mathcal{T}_{Q}^{K}(i, \boldsymbol{n}) \sum_{Q^{\prime}} N_{Q Q^{\prime}}^{K}(\boldsymbol{B})(-1)^{Q^{\prime}} \mathcal{T}_{-Q^{\prime}}^{K}\left(j, \boldsymbol{n}^{\prime}\right),
\end{aligned}
$$

where $\mathcal{T}_{Q}^{K}(i, \boldsymbol{n})$ are the irreducible spherical tensors for polarimetry introduced by Landi Degl'Innocenti (1984). The index $K$ takes the values $K=0,1,2$. The index $Q$ takes $(2 K+1)$ integer values in the range $-K \leq Q \leq+K$. For the lower index, we have followed the usual notation $Q$. There should be no confusion with the Stokes $Q$ parameter that never appears as an index in this paper. The indices $i, j$ refer to the Stokes parameters $(i, j=0, \ldots, 3)$. The coefficients $\mathcal{T}_{Q}^{K}(i, \boldsymbol{n})$ with $i=1,2$, associated to linear polarization, also depend on a reference angle, often denoted $\gamma$, needed to define the reference frame of the electric field in a plane perpendicular to $\boldsymbol{n}$ (see Fig. 5.14 in Landi Degl'Innocenti \& Landolfi 2004). Hanle effect measurements are usually performed close to the solar limb, with the spectrograph slit parallel to the nearest limb. Stokes $Q$ is negative along the slit (positive in the direction perpendicular to it) for $\gamma=0$. The elements $N_{Q Q^{\prime}}^{K}$ of the magnetic kernel depend on the magnetic field vector, on atomic parameters and collision rates (for details see Appendix A).

In this paper we consider a one-dimensional medium (planeparallel atmosphere). The direction of the magnetic field and of the radiation beams are reckoned in an atmospheric reference frame with the $z$-axis along the outward normal to the medium. The polar angles of the magnetic field direction are denoted by $\theta_{B}$ and $\chi_{B}$, and the polar angles of the directions $\boldsymbol{n}$ and $\boldsymbol{n}^{\prime}$ are denoted by $\theta, \chi$ and $\theta^{\prime}, \chi^{\prime}$ (see Fig. 1).

The random magnetic field $\boldsymbol{B}$ is modeled by a KuboAnderson process (KAP). It is a Markov process, discontinuous, stationary, and piecewise constant (Brissaud \& Frisch 1971, 1974). By definition, a random function $m(t)$ is a KAP, if the jumping times $t_{i}$ are uniformly and independently distributed in $[-\infty,+\infty]$ according to a Poisson distribution. Furthermore, $m(t)=m_{i}$ for $t_{i} \leq t \leq t_{i+1}$ where the $m_{i}$ are independent random variables with the same probability density $P(m)$. A KAP is thus fully characterized by a probability density $P(m)$ and a correlation time $t_{\text {cor }}=1 / v_{t}$, with $v_{t}$ the density of jumping times on the time axis (Papoulis 1965, p. 557). For a KAP, the covariance $\left\langle m(t) m\left(t^{\prime}\right)\right\rangle$ varies as $\mathrm{e}^{-v_{\mathrm{t}}\left|t-t^{\prime}\right|}$, this means that the spectrum is algebraic.

For the Hanle effect, polarization is created by a scattering process, which implies that the photons make a random walk inside the medium. If the magnetic field is a Markov process, say along the normal to a plane-parallel atmosphere, the radiation field at a point $\boldsymbol{r}$, depends on magnetic field values below and

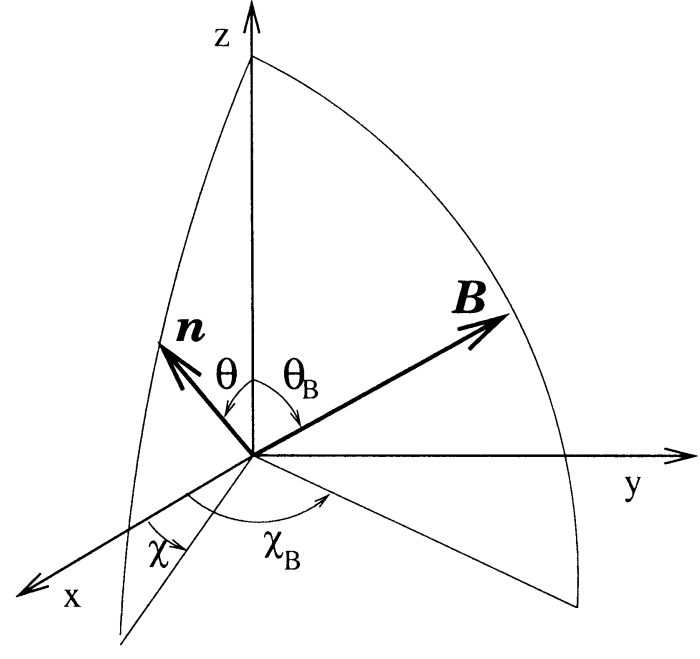

Fig. 1. Atmospheric reference frame with the definition of $(\theta, \chi)$ and $\left(\theta_{B}, \chi_{B}\right)$, the polar angles of the outgoing ray direction $\boldsymbol{n}$, and magnetic field vector $\boldsymbol{B}$. In the text we introduce the polar angles $\left(\theta^{\prime}, \chi^{\prime}\right)$ of the incoming ray direction $\boldsymbol{n}^{\prime}$.

above the point $\boldsymbol{r}$. To take advantage of the Markov character of the magnetic field, it is necessary to simplify a little and assume that the magnetic field is a random process in time, defined by a density $v_{\mathrm{t}}$ and a probability density $P(\boldsymbol{B})$. This approach was first used for random velocities with a finite correlation length by Frisch \& Frisch (1976). Its shortcoming is that it ignores correlations between photons that return to the same turbulent element after having been scattered a number of times (Frisch \& Frisch 1975). The Stokes vector $I$ then has to be taken as time dependent. Standard techniques of solutions for stochastic differential equations with Markov coefficients become applicable (Brissaud $\&$ Frisch 1974). They rely on the crucial remark that the joint random process in time $\{\boldsymbol{B}(t) ; \boldsymbol{I}(t)\}$ is also a Markov process. To simplify the notation we have omitted other independent variables on which the radiation field depends. As shown in HF06, the combination of the time-dependent transfer equation, with the evolution equation for the probability density of the joint process $\{\boldsymbol{B}(t) ; \boldsymbol{I}(t)\}$, provides a time-dependent transfer equation for a conditional mean Stokes vector $\boldsymbol{I}(t, \boldsymbol{r}, x, \boldsymbol{n} \mid \boldsymbol{B})$. For this radiation field, $\boldsymbol{B}$ plays the role of an additional independent variable with values distributed according to the probability density $P(\boldsymbol{B})$ (for the definition of the conditional mean see HF06).

The next step is to consider the stationary solution, $\boldsymbol{I}(\boldsymbol{r}, x, \boldsymbol{n} \mid \boldsymbol{B})$, for $t \rightarrow \infty$. It satisfies a transfer equation that has the usual advection, scattering, and primary source terms, but also contains an additional term describing the action of the magnetic field. Somewhat similar equations (without the scattering term) have been introduced for the Zeeman effect by Carroll \& Staude (2005). The mean Stokes parameters that one is looking for are given by

$\langle\boldsymbol{I}\rangle(\boldsymbol{r}, x, \boldsymbol{n})=\int P(\boldsymbol{B}) \boldsymbol{I}(\boldsymbol{r}, x, \boldsymbol{n} \mid \boldsymbol{B}) \mathrm{d}^{3} \boldsymbol{B}$.

In the next section we construct the stationary transfer equation for the conditional mean Stokes vector. We work with the irreducible components of the Stokes vector because they satisfy transfer equations that are simpler than the transfer equations for the Stokes parameters themselves. 


\section{The transfer problem}

We now concentrate on the case of a one-dimensional slab. We introduce the frequency averaged line optical depth $\tau$ defined by $\mathrm{d} \tau=-k(z) \mathrm{d} z$ with $z$ the coordinate along the vertical axis (see Fig. 1) and $k(z)$ the absorption coefficient per unit length. We denote by $T$ the total optical thickness of the slab with the surface at $\tau=0$ towards the observer. We assume that the incident radiation is zero on both sides of the slab.

For the deterministic Hanle effect with complete frequency redistribution, each component $S_{i}(\tau, \boldsymbol{n} ; \boldsymbol{B})$ of the emission term in the transfer equation (sum of the scattering and primary source terms) has an expansion of the form

$S_{i}(\tau, \boldsymbol{n} ; \boldsymbol{B})=\sum_{K Q} \mathcal{T}_{Q}^{K}(i, \boldsymbol{n}) S_{Q}^{K}(\tau ; \boldsymbol{B}), \quad i=0, \ldots 3$.

Starting from this expression, one can show (Frisch 2007, henceforth HF07) that the Stokes parameters have a similar expansion that can be written as

$I_{i}(\tau, x, \boldsymbol{n} ; \boldsymbol{B})=\sum_{K Q} \mathcal{T}_{Q}^{K}(i, \boldsymbol{n}) I_{Q}^{K}(\tau, x, \mu ; \boldsymbol{B}), \quad i=0, \ldots 3$,

where $\mu=\cos \theta$. Whereas the four Stokes parameters (three if one considers only linear polarization) depend on the two polar angles $\theta$ and $\chi$ defining $\boldsymbol{n}$, the nine irreducible components $I_{Q}^{K}$ (six only for linear polarization) are independent of the azimuthal angle $\chi$. This decomposition also holds for the conditional mean Stokes vector $I(\tau, x, \boldsymbol{n} \mid \boldsymbol{B})$ and the corresponding source vector $\boldsymbol{S}(\tau, \boldsymbol{n} \mid \boldsymbol{B})$. The components $I_{Q}^{K}$ and $S_{Q}^{K}$ can be regrouped into nine (or six) component vectors $\mathcal{I}(\tau, x, \mu \mid \boldsymbol{B})$ and $\mathcal{S}(\tau \mid \boldsymbol{B})$. We use calligraphic letters for the vectors $\mathcal{I}$ and $\mathcal{S}$ constructed with the $K Q$ decomposition and refer to them for simplicity as the "Stokes vector" and "source vector". We now give the transfer equation satisfied by $\mathcal{I}(\tau, x, \mu \mid \boldsymbol{B})$ in Sect. 3.1 and construct an integral equation for $\mathcal{S}(\tau \mid \boldsymbol{B})$ in Sect. 3.2. The symbols used in this paper are consistent with those used in HF06 and HF07.

\subsection{Transfer equation for the conditional mean Stokes parameters}

Proceeding as described in Sect. 2 (see also HF06), we find that $\mathcal{I}(\tau, x, \mu \mid \boldsymbol{B})$ satisfies the transfer equation

$$
\begin{aligned}
\mu \frac{\partial \mathcal{I}(\tau, x, \mu \mid \boldsymbol{B})}{\partial \tau}= & \varphi(x)[\mathcal{I}(\tau, x, \mu \mid \boldsymbol{B})-\mathcal{S}(\tau \mid \boldsymbol{B})] \\
& -v \int \Pi_{1}\left(\boldsymbol{B}, \boldsymbol{B}^{\prime}\right) \mathcal{I}\left(\tau, x, \mu \mid \boldsymbol{B}^{\prime}\right) \mathrm{d}^{3} \boldsymbol{B}^{\prime},
\end{aligned}
$$

where

$\mathcal{S}(\tau \mid \boldsymbol{B})=\boldsymbol{G}(\tau)+\hat{N}(\boldsymbol{B}) \mathcal{J}(\tau \mid \boldsymbol{B})$,

with

$$
\mathcal{J}(\tau \mid \boldsymbol{B})=\int_{-\infty}^{+\infty} \frac{1}{2} \int_{-1}^{+1} \varphi(x) \hat{\Psi}(\mu) \mathcal{I}(\tau, x, \mu \mid \boldsymbol{B}) \mathrm{d} \mu \mathrm{d} x .
$$

The operator $v \Pi_{1}$ describes the effects of the random magnetic field. The factor $v$ is now the mean number of jumping points per unit optical depth. It is related to the density of jumping times $v_{\mathrm{t}}$ by $v=v_{\mathrm{t}} / c k(z)$, with $c$ the speed of light. For simplicity we assume $v$ independent of $\tau$, but a depth-dependent $v$ could be handled (see e.g. Auvergne et al. 1973). Micro-turbulence corresponds to $v=\infty$ and macro-turbulence to $v=0$. Macro and micro-turbulence are also referred to as the optically thick and optically thin limits. The operator $\Pi_{1}$ is defined by

$\Pi_{1}=-\left[\delta\left(\boldsymbol{B}-\boldsymbol{B}^{\prime}\right)-P\left(\boldsymbol{B}^{\prime}\right)\right]$.

The matrix $\hat{N}(\boldsymbol{B})$ describes the Hanle effect. Construction rules for its elements $N_{Q Q^{\prime}}^{K}(\boldsymbol{B})$ are given in Eq. (A.4). When the magnetic field is zero, $\hat{N}(\boldsymbol{B})$ reduces to a diagonal matrix with elements depending only on the atomic model and collision rates (see Appendix A). The matrix $\hat{\Psi}(\mu)$ describes resonance polarization. Its elements $\Psi_{Q}^{K K^{\prime}}$ are real quantities. They can be found in LL04 (Appendix A20) or HF07 (see also Landi Degl'Innocenti et al. 1990). The primary source term $\mathcal{G}(\tau)$ is not random.

Averaging Eq. (6) over $P(\boldsymbol{B})$, we see that $\langle\mathcal{I}\rangle(\tau, x, \mu)$ satisfies the transfer equation

$\mu \frac{\partial\langle\mathcal{I}\rangle(\tau, x, \mu)}{\partial \tau}=\varphi(x)[\langle\mathcal{I}\rangle(\tau, x, \mu)-\langle\mathcal{S}\rangle(\tau)]$

where $\langle\boldsymbol{I}\rangle$ and $\langle\boldsymbol{S}\rangle$ are averages over the magnetic field vector PDF (see Eq. (3)). It is not possible to write an integral equation for $\langle\mathcal{S}\rangle(\tau)$ (except in the micro-turbulent limit). One must first calculate $\mathcal{S}(\tau \mid \boldsymbol{B})$ and then average it over $P(\boldsymbol{B})$.

\subsection{Integral equation for $\mathcal{S}(\tau \mid \mathbf{B})$}

With the boundary condition that there is no incident radiation on the outer surfaces of the slab, the formal solution of Eq. (6) can be written as

$$
\begin{aligned}
& \mathcal{I}(\tau, x, \mu \mid \boldsymbol{B})= \\
& \int_{\tau}^{T} \exp \left[-\frac{\tau^{\prime}-\tau}{\mu}\left(\varphi-v \Pi_{1}\right)\right] \varphi(x) \mathcal{S}\left(\tau^{\prime} \mid \cdot\right) \frac{\mathrm{d} \tau^{\prime}}{\mu} ; \mu>0 \\
& \mathcal{I}(\tau, x, \mu \mid \boldsymbol{B})= \\
& -\int_{0}^{\tau} \exp \left[-\frac{\tau^{\prime}-\tau}{\mu}\left(\varphi-v \Pi_{1}\right)\right] \varphi(x) \mathcal{S}\left(\tau^{\prime} \mid \cdot\right) \frac{\mathrm{d} \tau^{\prime}}{\mu} ; \mu<0 .
\end{aligned}
$$

The operator $\Pi_{1}$ acts on the variable denoted ".". It is standard notation for cases when the variable cannot be written explicitly. The action of $\Pi_{1}$ can be calculated by considering the Laplace transform

$\int_{0}^{\infty} \mathrm{e}^{-p E \varpi} \mathrm{e}^{\varpi v \Pi_{1}} \mathrm{~d} \varpi=\left(p E-v \Pi_{1}\right)^{-1}$,

where $\varpi=\left|\tau^{\prime}-\tau\right| / \mu$ and $E$ is the identity operator. To ensure convergence, $\mathfrak{R}(p)>0$. Solving for $f(\boldsymbol{B})$ the equation

$\left(p E-v \Pi_{1}\right) f(\boldsymbol{B})=g(\boldsymbol{B})$,

where $g(\boldsymbol{B})$ is known, one finds a simple expression that is easily expressed in terms of elementary Laplace transforms (for details see HF06; also Frisch \& Frisch 1976). We thus obtain

$$
\begin{gathered}
\mathcal{I}(\tau, x, \mu \mid \boldsymbol{B})=\int_{\tau}^{T} \varphi(x) \mathrm{e}^{-\frac{\tau^{\prime}-\tau}{\mu} \varphi(x)}\left\{\mathrm{e}^{-\frac{\tau^{\prime}-\tau}{\mu} v} \mathcal{S}\left(\tau^{\prime} \mid \boldsymbol{B}\right)\right. \\
\left.+\left[1-\mathrm{e}^{-\frac{\tau^{\prime}-\tau}{\mu} v}\right] \int P\left(\boldsymbol{B}^{\prime}\right) \mathcal{S}\left(\tau^{\prime} \mid \boldsymbol{B}^{\prime}\right) \mathrm{d}^{3} \boldsymbol{B}^{\prime}\right\} \frac{\mathrm{d} \tau^{\prime}}{\mu} ; \mu>0 \\
\mathcal{I}(\tau, x, \mu \mid \boldsymbol{B})=-\int_{0}^{\tau} \varphi(x) \mathrm{e}^{-\frac{\tau^{\prime}-\tau}{\mu} \varphi(x)}\left\{\mathrm{e}^{-\frac{\tau^{\prime}-\tau}{\mu} v} \mathcal{S}\left(\tau^{\prime} \mid \boldsymbol{B}\right)\right. \\
\left.+\left[1-\mathrm{e}^{-\frac{\tau^{\prime}-\tau}{\mu} v}\right] \int P\left(\boldsymbol{B}^{\prime}\right) \mathcal{S}\left(\tau^{\prime} \mid \boldsymbol{B}^{\prime}\right) \mathrm{d}^{3} \boldsymbol{B}^{\prime}\right\} \frac{\mathrm{d} \tau^{\prime}}{\mu} ; \mu<0 .
\end{gathered}
$$


The combination of Eqs. (15) and (16) with Eq. (7) yields the integral equation

$\mathcal{S}(\tau \mid \boldsymbol{B})=\boldsymbol{G}(\tau)+\hat{N}(\boldsymbol{B}) \Lambda[\mathcal{S}]$,

where

$$
\begin{aligned}
\Lambda[\mathcal{S}] & =\int_{0}^{T} \mathrm{~d} \tau^{\prime}\left\{\hat{\mathcal{L}}\left(\tau-\tau^{\prime} ; v\right) \mathcal{S}\left(\tau^{\prime} \mid \boldsymbol{B}\right)\right. \\
& \left.+\left[\hat{\mathcal{L}}\left(\tau-\tau^{\prime} ; 0\right)-\hat{\mathcal{L}}\left(\tau-\tau^{\prime} ; v\right)\right] \int P\left(\boldsymbol{B}^{\prime}\right) \mathcal{S}\left(\tau^{\prime} \mid \boldsymbol{B}^{\prime}\right) \mathrm{d}^{3} \boldsymbol{B}^{\prime}\right\}
\end{aligned}
$$

with

$$
\hat{\mathcal{L}}(\tau ; v)=\int_{-\infty}^{+\infty} \int_{0}^{1} \frac{1}{2 \mu} \hat{\Psi}(\mu) \mathrm{e}^{-\frac{|x|}{\mu}(\varphi(x)+v)} \varphi^{2}(x) \mathrm{d} \mu \mathrm{d} x .
$$

For $v=0$, we recover the usual kernel matrix for resonance scattering with complete frequency redistribution, denoted here by $\hat{K}(\tau)$ (see e.g. Landi Degl'Innocenti et al. 1990; Nagendra et al. 1998), and for $v=\infty$, we have $\hat{\mathcal{L}}(\tau ; v)=0$. Thus, in the micro-turbulent limit, the averaging of Eq. (17) over $P(\boldsymbol{B})$ yields a standard integral equation

$\langle\boldsymbol{S}\rangle(\tau)=\mathcal{G}(\tau)+\langle\hat{N}(\boldsymbol{B})\rangle \int_{0}^{T} \hat{K}\left(\tau-\tau^{\prime}\right)\langle\mathcal{S}\rangle\left(\tau^{\prime}\right) \mathrm{d} \tau^{\prime}$,

where $\langle\hat{N}(\boldsymbol{B})\rangle$ is the mean value of $\hat{N}(\boldsymbol{B})$, and $\hat{K}(\tau)=\hat{\mathcal{L}}(\tau ; 0)$.

\section{A PALI type numerical method of solution}

Several numerical methods of solution have been developed to solve integral equations arising in the study of the Hanle effect with a deterministic or micro-turbulent magnetic field. In Landi Degl'Innocenti et al. (1990), the system of linear integral equations for the components $\mathcal{S}_{Q}^{K}(\tau)$ is transformed into a system of linear equations for the $\mathcal{S}_{Q}^{K}\left(\tau_{i}\right)$ with $\tau_{i}$ the optical depth grid points. In this reference, the unknown functions are actually the density matrix elements $\rho_{Q}^{K}(\tau)$, but for a two-level atom with complete frequency redistribution, $\rho_{Q}^{K}(\tau)$ and $\mathcal{S}_{Q}^{K}(\tau)$ are proportional (see e.g. Landi Degl'Innocenti \& Bommier 1994).

Iterative methods of the ALI type have been developed for the Hanle effect with complete frequency redistribution (Nagendra et al. 1998; Manso Sainz \& Trujillo Bueno 1999, 2003) and partial frequency redistribution (Nagendra et al. 1999; Fluri et al. 2003; Sampoorna et al. 2008a). For partial frequency redistribution, the unknown functions depend on two independent variables: optical depth and frequency. Here we have a similar problem, the independent variables being now the optical depth and the magnetic field vector. We have developed a PALI method (P for polarized) described below to solve the integral Eq. (17) for $\mathcal{S}(\tau \mid \boldsymbol{B})$. The results are presented in Sect. 6.

We followed a standard approach by which one introduces an approximate $\Lambda$ operator denoted by $\Lambda^{*}$, choosing for $\Lambda^{*}$ the diagonal of $\Lambda$ with respect to optical depth. This is the so-called Jacobi scheme (Stoer \& Bulirsch 1983). It is the only one that has been used for partial frequency redistribution (see e.g. the review by Nagendra \& Sampoorna 2009, and references therein) and seemed to be an appropriate choice for exploratory work with random magnetic fields. More efficient iteration methods based on the Gauss-Seidel scheme have been developed for complete frequency redistribution (see e.g. Trujillo Bueno \& Fabiani Bendicho 1995; Léger et al. 2007).
The Jacobi iteration scheme is

$$
\begin{aligned}
{\left[\hat{E}-\hat{N}(\boldsymbol{B}) \Lambda^{*}\right] \delta \mathcal{S}^{(n)}(\tau \mid \boldsymbol{B}) } & = \\
\mathcal{G}(\tau) & +\hat{N}(\boldsymbol{B}) \mathcal{J}^{(n)}(\tau \mid \boldsymbol{B})-\mathcal{S}^{(n)}(\tau \mid \boldsymbol{B}),
\end{aligned}
$$

with

$\delta \boldsymbol{S}^{(n)}(\tau \mid \boldsymbol{B})=\boldsymbol{S}^{(n+1)}(\tau \mid \boldsymbol{B})-\boldsymbol{S}^{(n)}(\tau \mid \boldsymbol{B})$,

and

$\mathcal{J}^{(n)}(\tau \mid \boldsymbol{B})=\Lambda\left[\mathcal{S}^{(n)}\right]$

The superscript $(n)$ refers to the iteration step, and $\hat{E}$ is the identity matrix.

The righthand side in Eq. (21) is easy to calculate. Knowing $\mathcal{S}^{(n)}(\tau \mid \boldsymbol{B})$, one can calculate its mean value $\langle\boldsymbol{S}\rangle^{(n)}(\tau)$ by averaging over $P(\boldsymbol{B})$. Equations (15) and (16) are then used to calculate $\mathcal{I}(\tau, x, \mu \mid \boldsymbol{B})$. A short characteristic method (Kunasz \& Auer 1988; Auer \& Paletou 1994) is used for this step. Finally $\mathcal{J}^{(n)}(\tau \mid \boldsymbol{B})$ is deduced from Eq. (8).

Equation (18) shows that we only need the diagonal operator corresponding to $\mathcal{L}(\tau ; v)$, henceforth denoted $\mathcal{L}^{*}(\tau ; v)$, to construct the operator $\Lambda^{*}$. As Eq. (19) shows, it can be calculated by a standard method introduced in Auer \& Paletou (1994). At each grid point in space, we solve a transfer equation, like Eq. (10), where $\varphi(x)$ is replaced by $\varphi(x)+v$ and the source term replaced by a point source at the grid point under consideration. A short characteristic method is also used for this step. Finally, the elements of $\hat{\mathcal{L}}^{*}$ are obtained by performing the integration over $x$ and $\mu$ (see Eq. (19)).

The corrections $\delta \boldsymbol{S}^{(n)}(\tau \mid \boldsymbol{B})$ are solutions of Eq. (21). Since the operator $\Lambda^{*}$ is diagonal in space, there is no coupling between the different depth points. At each depth point $\tau_{q}$, we have a system of linear equations for $\delta \mathcal{S}^{(n)}\left(\tau_{q} \mid \boldsymbol{B}\right)$. The dimension of this system is $N_{B} \times N_{C}$, with $N_{C}$ the number of irreducible components ( 6 for linear polarization) and $N_{B}$ the number of grid points needed to describe the magnetic field PDF. Since the magnetic field is defined by its strength $B$, inclination $\theta_{B}$, and azimuth $\chi_{B}$ (see Fig. 1), $N_{B}=N_{B} \times N_{\theta_{B}} \times N_{\chi_{B}}$, with $N_{B}, N_{\theta_{B}}$, and $N_{\chi_{B}}$ the number of grid points corresponding to the respective variables.

At each depth point $\tau_{q}$, the linear system of equations for the $\delta \mathcal{S}_{j}^{(n)}$ can be written as

$$
\sum_{j} \hat{A}_{i j}\left(\tau_{q}\right) \delta \boldsymbol{S}_{j}^{(n)}\left(\tau_{q}\right)=\boldsymbol{r}_{i}^{(n)}\left(\tau_{q}\right)
$$

where $i$ and $j$ are indices for the magnetic field vector grid points $\left(i, j=1, \ldots, N_{\boldsymbol{B}}\right)$. The vectors $\delta \boldsymbol{S}_{j}^{(n)}$ and $\boldsymbol{r}_{i}^{(n)}$ have the dimension $N_{C}$. We use the notation $\delta \boldsymbol{S}_{j}^{(n)}\left(\tau_{q}\right)=\delta \boldsymbol{S}^{(n)}\left(\tau_{q} \mid \boldsymbol{B}_{j}\right)$. Similarly, $\boldsymbol{r}_{i}^{(n)}\left(\tau_{q}\right)=\boldsymbol{r}^{(n)}\left(\tau_{q} \mid \boldsymbol{B}_{i}\right)$. Each element $\hat{A}_{i j}$ is a $N_{C} \times N_{C}$ block given by

$$
\begin{aligned}
\hat{A}_{i j}\left(\tau_{q}\right)= & \delta_{i j} \hat{E} \\
& -\delta_{i j} \hat{N}_{i} \hat{\mathcal{L}}^{*}\left(\tau_{q} ; v\right)-\hat{N}_{i}\left[\hat{\mathcal{L}}^{*}\left(\tau_{q} ; 0\right)-\hat{\mathcal{L}}^{*}\left(\tau_{q} ; v\right)\right] \varpi_{j}
\end{aligned}
$$

The $\varpi_{j}$ are weights for the integration over the magnetic field PDF. The matrices $\hat{E}, \hat{N}_{i}=\hat{N}\left(\boldsymbol{B}_{i}\right)$, and $\hat{\mathcal{L}}^{*}\left(\tau_{q} ; v\right)$ corresponding to the operator $\mathcal{L}^{*}$, have the dimension $N_{C} \times N_{C}$. Explicit expressions for the elements of $\hat{N}$ and $\hat{\mathcal{L}}$ are given in Appendix A. The elements $A_{i j}$ have to be computed only once since they do not change during the iteration cycle. 
Table 1. A list of different PDFs used in this paper.

\begin{tabular}{ccc}
\hline \hline & $P_{\mathrm{S}}(B)$ & $P_{\mathrm{A}}\left(\theta_{B}\right)$ \\
\hline (i) & $P_{\mathrm{D}}(B)=\delta\left(B-B_{0}\right)$ & $P_{\text {iso }}\left(\theta_{B}\right)=\sin \theta_{B}$ \\
(ii) & $P_{\mathrm{M}}(B)=\frac{32}{\pi^{2} B_{0}}\left(B / B_{0}\right)^{2} \exp \left[-\frac{4}{\pi}\left(B / B_{0}\right)^{2}\right]$ & $P_{\mathrm{pl}-\mathrm{c}}\left(\theta_{B}\right)=(p+1)\left|\cos \theta_{B}\right|^{p} \sin \theta_{B}$ \\
(iii) & $P_{\mathrm{G}}(B)=\frac{2}{\pi B_{0}} \exp \left[-\frac{1}{\pi}\left(B / B_{0}\right)^{2}\right]$ & $P_{\mathrm{pl}-\mathrm{s}}\left(\theta_{B}\right)=\left(\sin \theta_{B}\right)^{p} \sin \theta_{B} / C_{p}$ \\
(iv) & $P_{\mathrm{E}}(B)=\frac{1}{B_{0}} \exp \left(-B / B_{0}\right)$ & \\
\hline
\end{tabular}

The convergence properties of this iteration method are similar to those of other PALI methods used for polarized problems (Nagendra et al. 1998, 1999). The new feature here is the discretization of the magnetic field vector. Typically we have been using $N_{B}=40$. For an isotropic angular distribution, $N_{\theta_{B}}=5-7$ points in the interval $[0, \pi]$, the integration over $\theta_{B}$ being performed with a Gauss-Legendre quadrature. Significantly higher values of $N_{\theta_{B}}$ are needed for angular distributions that are peaked along some direction (see Sect. 5). All the magnetic field PDFs chosen here have a cylindrical symmetry about the normal to the atmosphere, so no integration over $\chi_{B}$ is needed. For the integration over $\tau$, we use 5 to 7 points per decade.

In this work, we consider self-emitting slabs. The primary source is $G(\tau)=\epsilon B_{v_{0}} /(1+\epsilon)$ with $\epsilon$ the rate of destruction by inelastic collisions (see Appendix A) and $B_{v_{0}}$ the Planck function at line center. The line absorption profile $\varphi(x)$ is a Voigt function with damping parameter $a$. The atomic and atmospheric models are thus defined by a set of parameters $\left(T, a, \epsilon^{\prime}, B_{v_{0}}\right)$ where $a$, $\epsilon^{\prime}=\epsilon /(1+\epsilon)$ and $B_{v_{0}}$ are assumed to be constant with $\tau$. The solution of the transfer equation is then symmetrical with respect to $T / 2$.

The magnetic kernel elements $N_{Q Q^{\prime}}^{K}(\boldsymbol{B})$ are defined in Appendix A. In all the calculations we assume a normal Zeeman triplet, an electric-dipole transition and no depolarizing collisions. For the magnetic field, the parameters are the magnetic field strength $B$, the polar angles $\theta_{B}$ and $\chi_{B}$, the density $v$ of jumping points and the PDF $P(\boldsymbol{B})$. For the Hanle effect, it is convenient to use the Hanle efficiency factor $\Gamma_{B}$, instead of the magnetic field strength itself. The definition of $\Gamma_{B}$ is recalled in Appendix A.

\section{A choice of magnetic field vector PDFs}

For the quiet Sun, a few PDFs have been proposed in the literature for field strength $B$ and for inclination $\theta_{B}$ of the magnetic field with respect to the vertical direction. They are based on the analysis of magneto-convection simulations, inversion of Stokes parameters, and heuristic considerations (see e.g. Trujillo Bueno et al. 2004; Dominguez Cerdeǹa et al. 2006; Sánchez Almeida 2007; Sampoorna et al. 2008b). Almost nothing is known about the azimuthal distribution. For our investigation we have chosen PDFs that are cylindrically symmetrical and have the form

$$
\begin{aligned}
P(\boldsymbol{B}) \mathrm{d}^{3} \boldsymbol{B} & =f(B) g\left(\theta_{B}\right) B^{2} \sin \theta_{B} \mathrm{~d} B \mathrm{~d} \theta_{B} \frac{\mathrm{d} \chi_{B}}{4 \pi}, \\
0 & \leq B<+\infty, \quad \theta_{B} \in[0, \pi], \quad \chi_{B} \in[0,2 \pi] .
\end{aligned}
$$

For convenience, we rewrite them as

$P(\boldsymbol{B}) \mathrm{d}^{3} \boldsymbol{B}=\frac{1}{2} P_{\mathrm{S}}(B) P_{\mathrm{A}}\left(\theta_{B}\right) \mathrm{d} B \mathrm{~d} \theta_{B}$.

Our choices for the strength and angular distributions are presented in Table 1.

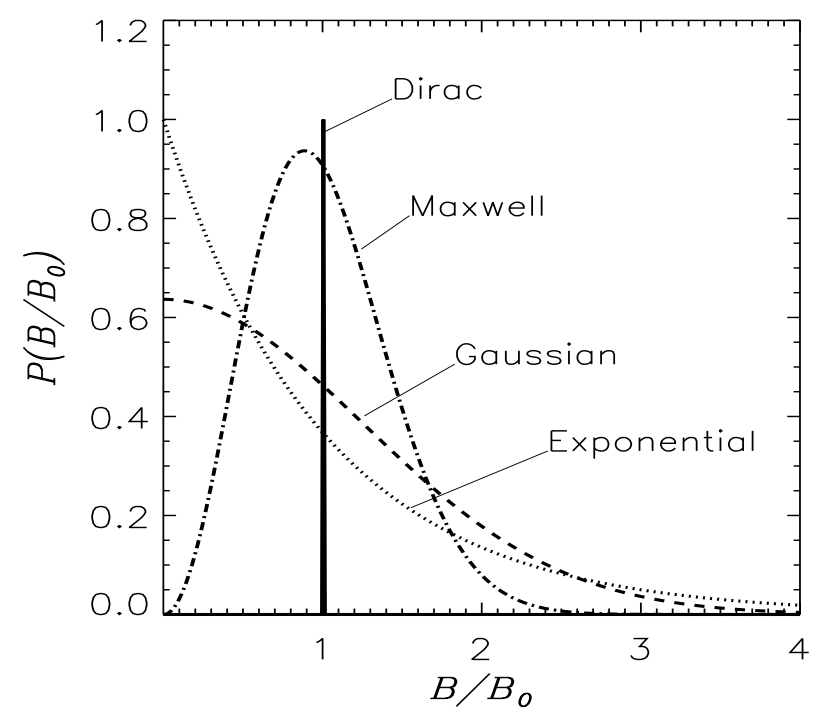

Fig. 2. Probability density functions $P\left(B / B_{0}\right)$ as a function of $\left(B / B_{0}\right)$. Solid line: $P_{\mathrm{D}}\left(B / B_{0}\right)$; dotted line: $P_{\mathrm{E}}\left(B / B_{0}\right)$; dashed line: $P_{\mathrm{G}}\left(B / B_{0}\right)$; dot-dashed line: $P_{\mathrm{M}}\left(B / B_{0}\right)$.

For $P_{\mathrm{S}}(B)$, we have chosen a delta function, $P_{\mathrm{D}}(B)$, an exponential distribution, $P_{\mathrm{E}}(B)$, a Gaussian distribution, $P_{\mathrm{G}}(B)$, and a Maxwell Distribution, $P_{\mathrm{M}}(B)$. They are plotted in Fig. 2 as a function of $B / B_{0}$. These functions are normalized to unity. They have the same mean value, $\langle B\rangle=B_{0}$, but the variance $\sigma=\left[\left\langle B^{2}\right\rangle-\langle B\rangle^{2}\right]^{1 / 2}$ changes : for the exponential distribution, $\sigma=B_{0}$, for the Gaussian distribution, $\sigma=\sqrt{\pi / 2} B_{0}$, and for the Maxwell distribution, $\sigma=[(3 \pi / 8)-1]^{1 / 2} B_{0}$.

For the angular distribution (see Table 1, second column), we have retained the isotropic distribution $P_{\text {iso }}$, frequently used in the analysis of the Hanle effect. It was introduced by Stenflo (1982) to model weak magnetic fields that are passively tangled by the turbulent motions (see also Stenflo 2009).

Recent Hinode observations suggest a predominantly horizontal magnetic flux in the quiet Sun (Lites et al. 2008). This finding is supported by some numerical simulations (Schüssler $\&$ Vögler 2008). This type of distribution can be modeled with the sine power law $P_{\mathrm{pl-s}}$, where $p(p \geq 0)$ is an index that can be chosen arbitrarily, and $C_{p}$ a normalization constant. When $p$ goes to zero, one recovers the isotropic distribution, and when $p$ goes to infinity, a purely horizontal random field, considered in Stenflo (1982). When $p$ is an integer, the normalization constant $C_{p}$ can be calculated explicitly. For even values of $p$,

$C_{p}=\frac{p \times(p-2) \times \cdots \times 2}{(p+1) \times(p-1) \times \cdots \times 3}$,

and for odd values of $p$,

$C_{p}=\frac{p \times(p-2) \times \cdots \times 1}{(p+1) \times(p-1) \times \cdots \times 2} \frac{\pi}{2}$. 


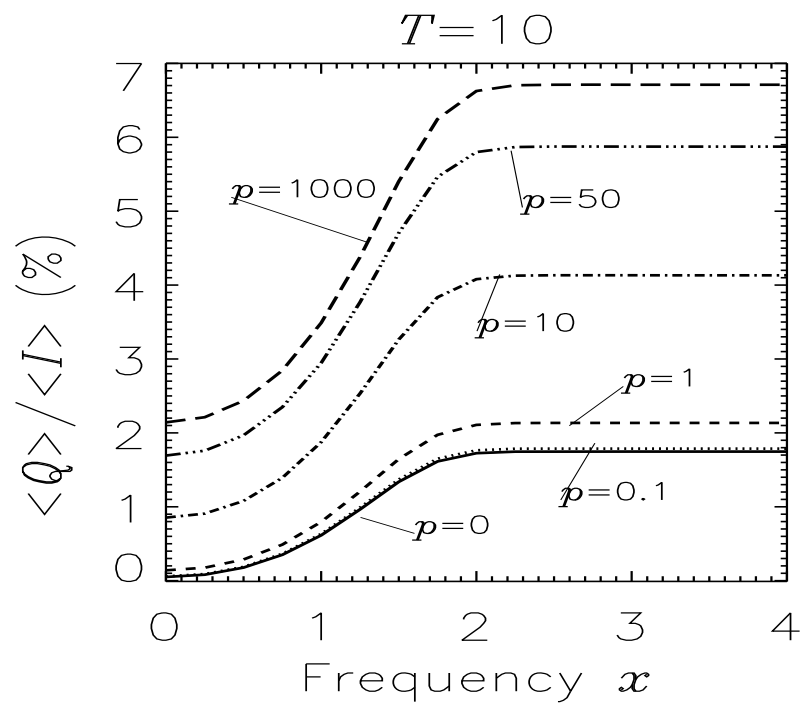

Fig. 3. Effect of the cosine power-law index $p$ on $\langle Q\rangle /\langle I\rangle$ at $\tau=0$, $\mu=0.05$, for the model parameters $\left(T, a, \epsilon^{\prime}, B_{v_{0}}\right)=\left(10^{4}, 10^{-3}, 10^{-4}, 1\right)$, and in the micro-turbulent limit. Solid line: $p=0$; dotted line: $p=0.1$; dashed line: $p=5$; dot-dashed line: $p=50$, and dash-triple-dotted line: $p=1000$.

When $p=0$, we have $C_{p}=1$. When $p$ goes to infinity, $C_{p}$ goes to zero. Setting $p=2 m$ for even values of $p$, and $p=2 m-1$ for odd values $(m \geq 1)$, one can establish that $C_{p} \rightarrow \sqrt{\pi / m} / 2$ when $m \rightarrow \infty$.

The cosine power law $P_{\mathrm{pl}-\mathrm{c}}$ was introduced in Stenflo (1987) to investigate the Zeeman effect with random magnetic fields that may become predominantly vertical. It was used in Sampoorna et al. (2008b) to construct a composite PDF that mimics a distribution becoming more and more vertical as the field strength increases. When $p=0$, the distribution is isotropic. When $p$ increases the field becomes more and more vertical. In the limit $p \rightarrow \infty$, the Hanle effect disappears because the scattering atoms are illuminated by an unpolarized field, cylindrically symmetrical about the magnetic field direction. This effect is illustrated in Fig. 3. We see that the ratio $\langle Q\rangle /\langle I\rangle$ increases with $p$. It reaches the Rayleigh limit when $p=1000$. The mean Stokes parameters, $\langle Q\rangle$ and $\langle I\rangle$, have been calculated in the micro-turbulent limit, for a magnetic field with constant strength, corresponding to a Hanle factor $\Gamma_{\mathrm{B}_{0}}=1$.

\section{Dependence of the polarization on the correlation length}

To examine the dependence of the polarization on the correlation length $1 / v$ (in Doppler width units), we examined the surface value of the ratio $\langle Q\rangle /\langle I\rangle$ at the limb $(\mu=0.05),\langle Q\rangle$ and $\langle I\rangle$ being the mean values of Stokes $Q$ and $I$, for several values of $v$ and $T$.

We first chose the simplest PDF, namely an isotropic angular distribution with a Dirac distribution $\delta\left(B-B_{0}\right)$. The parameter $\Gamma_{\mathrm{B}_{0}}$ was set to unity. We found that the dependence of $\langle Q\rangle /\langle I\rangle$ on the value of $v$ is quite weak for optically thin $(T \ll 1)$ lines, and also optically thick $\left(T \geq 10^{3}\right)$ ones. For lines with a moderate optical depth $(T=10)$, some dependence could be observed, the maximum variation of the ratio $\langle Q\rangle /\langle I\rangle$ being about $0.1 \%$.

Keeping the assumption of a single value field strength, we calculated the ratio $\langle Q\rangle /\langle I\rangle$ for the sine and cosine power law distributions (see Table 1). For the sine power law, we chose $p=50$. For this value of $p$, the distribution is strongly peaked in

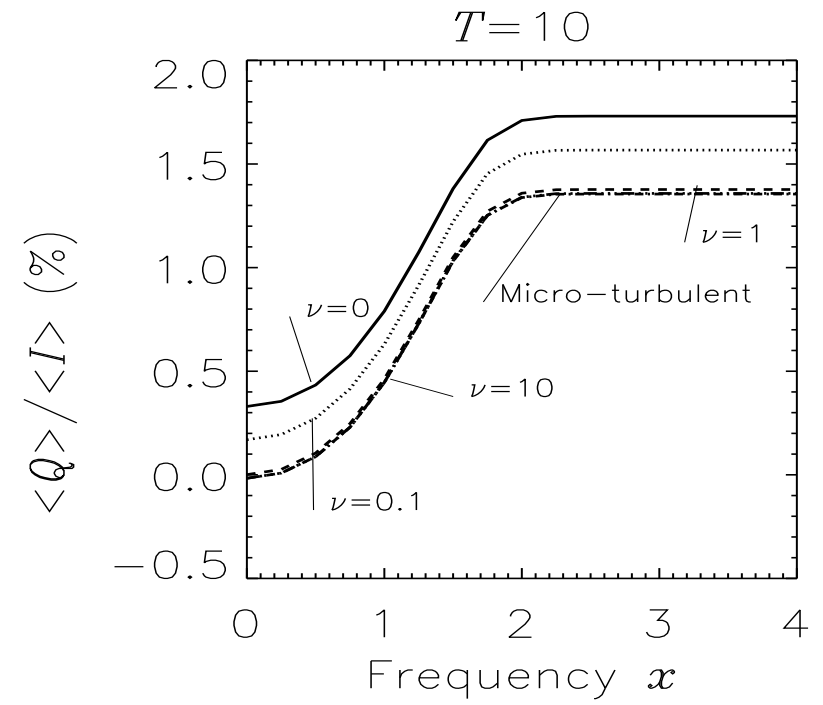

Fig. 4. Dependence of $\langle Q\rangle /\langle I\rangle$ on the correlation length $1 / v$. Sine power law angular distribution with single value field strength $\left(\Gamma_{B_{0}}=1\right)$. Slab with an optical thickness $T=10$. Solid line: $v=0$; dotted line: $v=0.1$; dashed line: $v=1$; dot-dashed: $v=10$, and triple-dot dashed: micro.

the horizontal direction. For the cosine power law, we retained $p=5$. The distribution is also strongly peaked, but in the vertical direction (see Fig. 11 in Sampoorna et al. 2008b) and the diminution of the Hanle effect is significant (see Fig. 3). For these two distributions, the dependence on the correlation length is also negligible for optically thin and optically thick lines. Some dependence appears for lines with an intermediate optical depth. Figure 4, corresponding to the sine power law and $T=10$, shows that the difference $(\langle Q\rangle /\langle I\rangle)_{\text {macro }}-(\langle Q\rangle /\langle I\rangle)_{\text {micro }} \simeq 0.3 \%$ all along the polarization profile. The variation in $\langle Q\rangle /\langle I\rangle$ is coming almost exclusively from the variation in $\langle Q\rangle$, since the dependence of Stokes $I$ on the magnetic field is very small for the Hanle effect. This figure also shows that the micro-turbulent limit is reached for $v \simeq 10$. The reason is that $v$ only enters in exponential terms, as can be seen in Eq. (19). For the cosine power law and $T=10$, we found a very similar behavior to that shown in Fig. 4, but the polarization is somewhat stronger because of the reduction of the Hanle effect.

To understand the dependence on the correlation length, we examined the dependence on $v$ and $\theta_{B}$ of the conditional source function component $S_{0}^{2}\left(\tau=0 \mid B, \theta_{B}\right)$. This function depends strongly on $v$ and $\theta_{B}$, with the micro and macro-turbulent limits showing quite different variation with $\theta_{B}$. The averaging over $\theta_{B}$ eliminates most of the variation with $v$. Some of it may remain, however, in particular when the angular distribution is peaked in the horizontal or vertical direction.

A very low sensitivity to the value of the correlation length is a strong indication that the polarization is created locally. For a line with a very small optical thickness, $T \ll 1$, photons will suffer about one scattering and the polarization is well represented by the so-called single scattering approximation. For very thick lines, although photons suffer a very large number of scattering events, the polarization is created near the surface by a few of them. In these two limits, the polarization thus cannot feel the correlation length of the magnetic field. For $T=10$, we have an intermediate situation with a clear sensitivity to the correlation length.

For the Hanle effect, the polarization can be evaluated by a perturbation method leading to a series expansion in terms of a mean number of scattering events (see HF06). In the next section 
we show how to construct this expansion. We use it to examine how many terms are needed to reproduce the exact solution and thus give a somewhat quantitative content to the above remarks.

\section{A series expansion for the calculation of the polarization}

The construction of a series expansion for the calculation of the polarization is possible for the following three reasons: (i) the Hanle polarization is weak; (ii) it is controlled by the anisotropy of the radiation field; (iii) at each scattering a significant amount of polarization is being lost. This last point will be clarified below.

Here, for simplicity we present the perturbation method and discuss its convergence properties for the simple case of a deterministic (or micro-turbulent) magnetic field. We then show how to carry it out for magnetic fields with a finite correlation length and propose a perturbation expansion that is an improved version of the method presented in HF06.

\subsection{Construction of the expansion}

We start from the standard integral equation for the Hanle effect with a deterministic magnetic field, namely

$\mathcal{S}(\tau ; \boldsymbol{B})=\boldsymbol{G}(\tau)+\hat{N}(\boldsymbol{B}) \int_{0}^{T} \hat{K}\left(\tau-\tau^{\prime}\right) \mathcal{S}\left(\tau^{\prime} ; \boldsymbol{B}\right) \mathrm{d} \tau^{\prime}$

In the micro-turbulent limit, $\hat{N}(\boldsymbol{B})$ should be replaced by its mean value over the magnetic field PDF and $\mathcal{S}$ will depend only on $\tau$. With $\boldsymbol{B}=0$, this equation describes the Rayleigh scattering.

In the deterministic case, if the magnetic field is a constant, the dependence on the azimuthal angle $\chi_{B}$ can be factored out as shown in Appendix A. Henceforth we work with the components $S_{Q}^{K}=\mathrm{e}^{\mathrm{i} Q \chi_{B}} \mathcal{S}_{Q}^{K}$ and to simplify the notation, the dependence on $\boldsymbol{B}$ is omitted. These new components satisfy the set of equations $S_{Q}^{K}(\tau)=\delta_{K 0} \delta_{Q 0} G(\tau)$

$$
+\sum_{K^{\prime} Q^{\prime}} N_{Q Q^{\prime}}^{K}(\boldsymbol{B}) \int_{0}^{T} K_{Q^{\prime}}^{K K^{\prime}}\left(\tau-\tau^{\prime}\right) S_{Q^{\prime}}^{K^{\prime}}\left(\tau^{\prime}\right) \mathrm{d} \tau^{\prime},
$$

with $K_{Q^{\prime}}^{K K^{\prime}}(\tau)$ the components of the matrix $\hat{K}(\tau)$. The notation $\boldsymbol{B}$ now stands for $\left(B, \theta_{B}\right)$. The components $I_{Q}^{K}$ of the radiation field satisfy the transfer equation

$\mu \frac{\partial I_{Q}^{K}(\tau, x, \mu)}{\partial \tau}=\varphi(x)\left[I_{Q}^{K}(\tau, x, \mu)-S_{Q}^{K}(\tau)\right]$.

We first consider the equation for $S_{0}^{0}$. Only $S_{0}^{2}$ appears in the righthand side since $K=0$ implies $Q=Q^{\prime}=0$. For the Hanle effect, the polarization is always weak and its effect on Stokes I may be neglected, at least in a first approximation. Neglecting the contribution from $S_{0}^{2}$, we obtain

$\tilde{S}_{0}^{0}(\tau)=G(\tau)+N_{00}^{0} \int_{0}^{T} K_{0}^{00}\left(\tau-\tau^{\prime}\right) \tilde{S}_{0}^{0}\left(\tau^{\prime}\right) \mathrm{d} \tau^{\prime}$

The notation $\tilde{S}_{Q}^{K}$ is used to denote approximate values. Equation (33) is the usual unpolarized integral equation for the source function where $N_{00}^{0}=1 /(1+\epsilon)$.
We now replace $S_{0}^{0}$ by $\tilde{S}_{0}^{0}$ in the equation for $S_{Q}^{2}$ and obtain

$$
\begin{aligned}
\tilde{S}_{Q}^{2}(\tau)= & N_{Q 0}^{2}(\boldsymbol{B}) C_{0}^{2}(\tau) \\
& +\sum_{Q^{\prime}} N_{Q Q^{\prime}}^{2}(\boldsymbol{B}) \int_{0}^{T} K_{Q^{\prime}}^{22}\left(\tau-\tau^{\prime}\right) \tilde{S}_{Q^{\prime}}^{2}\left(\tau^{\prime}\right) \mathrm{d} \tau^{\prime}
\end{aligned}
$$

where

$C_{0}^{2}(\tau)=\int_{0}^{T} K_{0}^{20}\left(\tau-\tau^{\prime}\right) \tilde{S}_{0}^{0}\left(\tau^{\prime}\right) \mathrm{d} \tau^{\prime}$

The kernel $K_{0}^{20}(\tau)$ is sometimes denoted $K_{12}(\tau)$ (e.g. Landi Degl'Innocenti et al. 1990; Nagendra et al. 1998). Its integral over $\tau$ in the interval $[0,+\infty]$ is zero. The function $C_{0}^{2}(\tau)$, can also be written as

$C_{0}^{2}(\tau)=\int_{-\infty}^{+\infty} \frac{1}{2} \int_{-1}^{+1} \Psi_{0}^{20}(\mu) \varphi(x) \tilde{I}_{0}^{0}(\tau, x, \mu) \mathrm{d} \mu \mathrm{d} x$,

with $\Psi_{0}^{20}(\mu)=\frac{3}{2 \sqrt{2}}\left(3 \mu^{2}-1\right)$. In this form we recognize the dominant term in the radiation spherical tensor $\vec{J}_{0}^{2}(\tau)$. This function, which is zero for an isotropic radiation field, serves to measure the anisotropy of the field (see e.g. Trujillo Bueno 2001, LL04).

Equation (34) shows that $N_{Q 0}^{2}(\boldsymbol{B}) C_{0}^{2}(\tau)$ is the driving term for the polarization. This suggests solving this equation by the standard method of successive iterations for Fredholm integral equations of the second type (Iyanaga \& Kawada 1970). For radiative transfer problems, this method is usually referred to as $\Lambda$-iteration. The zeroth-order solution in this iteration scheme is given by $N_{Q 0}^{2}(\boldsymbol{B}) C_{0}^{2}(\tau)$. The recurrence scheme may be written as

$$
\begin{aligned}
{\left[\tilde{S}_{Q}^{2}\right]^{(k)}=} & N_{Q 0}^{2}(\boldsymbol{B}) C_{0}^{2}(\tau) \\
& +\sum_{Q^{\prime}} N_{Q Q^{\prime}}^{2}(\boldsymbol{B}) \int_{0}^{T} K_{Q^{\prime}}^{22}\left(\tau-\tau^{\prime}\right)\left[\tilde{S}_{Q^{\prime}}^{2}\left(\tau^{\prime}\right)\right]^{(k-1)} \mathrm{d} \tau^{\prime}
\end{aligned}
$$

with $\left[\tilde{S}_{Q}^{2}\right]^{(0)}=N_{Q 0}^{2}(\boldsymbol{B}) C_{0}^{2}(\tau)$.

It is well known that the $\Lambda$-iteration applied to Eq. (33) has a very poor convergence rate when $T$ is large and $\epsilon$ very small, because the kernel $K_{0}^{00}$ is normalized to unity and the coefficient $N_{00}^{0}$ almost equal to unity. In Eq. (34) the situation is radically different because the kernels $K_{Q}^{22}(\tau)$ have integrals over $[-\infty,+\infty]$ which are less than unity, actually they are all equal to 7/10 (see e.g. HF06), and the coefficients $N_{Q 0}^{2}(\boldsymbol{B})$ are also significantly smaller than unity when $\boldsymbol{B}$ is not zero. For Rayleigh scattering, the only non-zero coefficient is $N_{00}^{2}$, which is close to the depolarization parameter $W_{K}\left(J_{1}, J_{\mathrm{u}}\right)$ (see Appendix A).

To examine the convergence properties of this iteration scheme, we can consider a simplified version of Eq. (34). The righthand side of this equation contains a driving term, a transport term corresponding to $Q^{\prime}=Q$, and terms coupling $\tilde{S}_{Q}^{2}$ with the $\tilde{S}_{Q^{\prime}}^{2}, Q^{\prime} \neq Q$. Neglecting these last terms, we see that the solution at step $(k)$ can be written as a series expansion of the form

$$
\begin{aligned}
{\left[\tilde{S}_{Q}^{2}\right]^{(k)}=} & N_{Q 0}^{2}(\boldsymbol{B}) C_{0}^{2}(\tau)+\sum_{m=1}^{m=k}\left[\frac{7}{10} N_{Q Q}^{2}\right]^{m} \\
& \times \int_{0}^{T} \bar{K}_{Q}^{22}\left(\tau-\tau_{1}\right) \mathrm{d} \tau_{1} \int_{0}^{T} \bar{K}_{Q}^{22}\left(\tau_{1}-\tau_{2}\right) \mathrm{d} \tau_{2} \ldots \\
& \times \int_{0}^{T} \bar{K}_{Q}^{22}\left(\tau_{k-1}-\tau_{k}\right) N_{Q 0}^{2}(\boldsymbol{B}) C_{0}^{2}\left(\tau_{k}\right) \mathrm{d} \tau_{k}
\end{aligned}
$$


Here the kernels $\bar{K}_{Q}^{22}$, defined by $\bar{K}_{Q}^{22}=\frac{10}{7} K_{Q}^{22}$, are normalized to unity. The term of order $m$ contains the contribution of all the photons that have been scattered $(m+1)$ times, the first scattering corresponding to the creation of the primary source $N_{Q 0}^{2}(\boldsymbol{B}) C_{0}^{2}(\tau)$. Since the kernels $\bar{K}_{Q}^{22}$ are positive and normalized to unity, the ratio of the term of order $(m+1)$ over the term of order $m$ scales as $0.7 N_{Q Q}^{2}(\boldsymbol{B})$. Since the $N_{Q Q}^{2}(\boldsymbol{B})$ are less than unity, one can expect that a few terms in the series will suffice to provide a good approximation to the exact solution. Somewhat more accurate predictions can be made for optically thin and optically thick lines.

For optically thin lines $(T \ll 1)$, one can approximate $\left[\tilde{S}_{Q}^{2}\right]^{(k)}(\tau)$ by

$\left[\tilde{S}_{Q}^{2}\right]^{(k)}(\tau) \simeq N_{Q 0}^{2}(\boldsymbol{B}) C_{0}^{2}(\tau)\left[1+\sum_{m=1}^{k}\left[\frac{7}{10} N_{Q Q}^{2}(\boldsymbol{B})\right]^{m} T^{m}\right]$.

The driving term is dominant and suffices to correctly evaluate the polarization. This is the so-called single scattering approximation.

To examine the case of optically thick lines, we can let $T \rightarrow$ $\infty$. If we approximate $\bar{K}_{Q}^{22}(\tau)$ by a delta function, we obtain

$\left[\tilde{S}_{Q}^{2}\right]^{(k)}(\tau) \simeq N_{Q 0}^{2}(\boldsymbol{B}) C_{0}^{2}(\tau)\left[1+\sum_{m=1}^{k}\left[\frac{7}{10} N_{Q Q}^{2}(\boldsymbol{B})\right]^{m}\right]$.

This expression shows that a single scattering can also provide a reasonable approximation for optically thick lines. We also see that the smaller $N_{Q Q}^{2}(\boldsymbol{B})$, the better the single scattering approximation and the faster the speed of convergence of the series expansion. We also note that the $N_{Q Q}^{2}(\boldsymbol{B})$ are positive, hence the sum inside the square brackets increases with the value of $k$.

For lines with very large optical thicknesses, the value of Stokes $Q$ at the surface can be easily related to $S_{0}^{2}(\tau)$. For these lines, $Q$ is controlled by the component $I_{0}^{2}$. Using $\mathcal{T}_{0}^{2}(1, \boldsymbol{n})=$ $-3\left(1-\mu^{2}\right) /(2 \sqrt{2})$ for $\gamma=0$, and the Eddington-Barbier relation, we obtain

$Q(0, x, \mu) \simeq-\frac{3}{2 \sqrt{2}}\left(1-\mu^{2}\right) \tilde{S}_{0}^{2}\left(\frac{\mu}{\varphi(x)}\right)$.

We have performed a few numerical experiments described below to give a quantitative proof to these predictions.

\subsection{Numerical results}

The computation of the polarization by the series expansion method involves the following steps:

(i) solution of Eq. (33) for $\tilde{S}_{0}^{0}$ by an ALI method and calculation of the corresponding scalar radiation field $\tilde{I}_{0}^{0}$;

(ii) computation of $C_{0}^{2}(\tau)$ with Eq. (36);

(iii) calculation of the source terms $\left[\tilde{S}_{Q}^{2}\right]^{(k)}$, with the iterative scheme in Eq. (37), starting from $N_{Q 0}^{2}(\boldsymbol{B}) C_{0}^{2}(\tau)$;

(iv) at each step $(k)$, solution of Eq. (32) by a short characteristic method, calculation of the Stokes parameters with Eq. (5), and of the ratio

$r^{(k)}=\left[\left|p^{(k)}-p^{(k-1)}\right|\right] / p^{(k)}$,

at $\tau=0, x=0, \mu=0.05$. Here $p=\left\{[Q / I]^{2}+[U / I]^{2}\right\}^{1 / 2}$.

The iterations are stopped when $r^{(k)}<10^{-3}$.
Table 2. Number of iterations needed to reproduce the exact solution with a relative error about $10^{-3}$ at line center, with the parameters of the magnetic field in Cols. 2 and 3 the same as in Figs. 6 and 7.

\begin{tabular}{cccc}
\hline \hline & Rayleigh & Deterministic & Micro-turbulent \\
$T$ & $N_{k}$ & $N_{k}$ & $N_{k}$ \\
\hline $10^{-2}$ & 3 & 3 & 3 \\
$10^{-1}$ & 4 & 4 & 4 \\
1 & 7 & 7 & 5 \\
10 & 16 & 16 & 9 \\
$10^{2}$ & 12 & 11 & 4 \\
$10^{3}$ & 7 & 6 & 5 \\
$10^{4}$ & 7 & 7 & 5 \\
$10^{6}$ & 8 & 7 & 5 \\
$10^{8}$ & 8 & 7 & 5 \\
\hline
\end{tabular}

The polarization has been calculated by this expansion method for several values of the slab optical thickness $T$ varying between $10^{-2}$ and $10^{8}$. For each value of $T$, we considered the Rayleigh scattering, a deterministic magnetic field, and a micro-turbulent magnetic field. For the deterministic case, we chose $\Gamma_{B}=1$, $\theta_{B}=30^{\circ}$, and $\chi_{B}=45^{\circ}$. For the micro-turbulent case, the magnetic field has an isotropic angular distribution and takes a single value $B_{0}$, with $\Gamma_{B_{0}}=1$. The coefficients $N_{Q Q^{\prime}}^{2}$ are replaced by their mean values over the isotropic distribution. In each case, the exact solution is calculated with a PALI method applied to Eq. (31). For Rayleigh scattering and the micro-turbulent magnetic field, Stokes $U$ is zero and Stokes $Q$ depends only on the inclination angle $\theta$ of the line of sight (see Fig. 1). For a deterministic magnetic field, the Stokes parameters $Q$ and $U$ also depend on the azimuthal angle $\chi$. In the calculations presented here $\chi=0$.

We show in Table 2 the number $N_{k}$ of iterations defined by the criterion $r^{(k)}<10^{-3}$. We stress that the value of $N_{k}$ has nothing to do with the number of iterations of the PALI method, the latter being controlled by the choice of the approximate $\Lambda^{*}$ operator. In Figs. 5 to 7 we show the results of our calculations for $T=10$ and $T=10^{4}$, Fig. 5 being devoted to the Rayleigh scattering, Fig. 6 to the deterministic Hanle effect, and Fig. 7 to the micro-turbulent case. In each panel we plotted the exact values of $Q / I$ and a few iteration steps. In the micro-turbulent case, we plotted $\langle Q\rangle /\langle I\rangle$.

We observe that the series expansion properly converges to the exact solution, that single scattering provides an approximation that is much better for $T=10^{4}$ than for $T=10$, and that the accuracy of this approximation improves from Rayleigh scattering to a deterministic and micro-turbulent Hanle effect. These last two points are illustrated in Fig. 8 where we show the difference

$e_{\mathrm{ss}}=\left(Q_{\text {exact }}-Q_{\mathrm{ss}}\right) / I$,

calculated at $\tau=0, x=0, \mu=0.05$, as a function of the slab optical thickness $T$. Here, $Q_{\text {exact }}$ is the solution of Eq. (30), $Q_{\text {ss }}$ is given by the single scattering approximation, and $I$ is the exact value of Stokes $I$. We see that for $T$ small, $e_{\mathrm{ss}}$ increases with $T$ in agreement with Eq. (39). For large $T$, it becomes essentially independent of $T$ as predicted by Eq. (40). It goes through a maximum around $T=10$.

The decrease in $e_{\mathrm{ss}}$ from Rayleigh scattering to microturbulent Hanle effect, is directly related to the value of the elements $N_{Q Q}^{2}$. For Rayleigh scattering, the index $Q$ takes only the value $Q=0$ and $N_{00}^{2}=1 /(1+\epsilon)$ (assuming $\left.W_{K}=1\right)$. For 

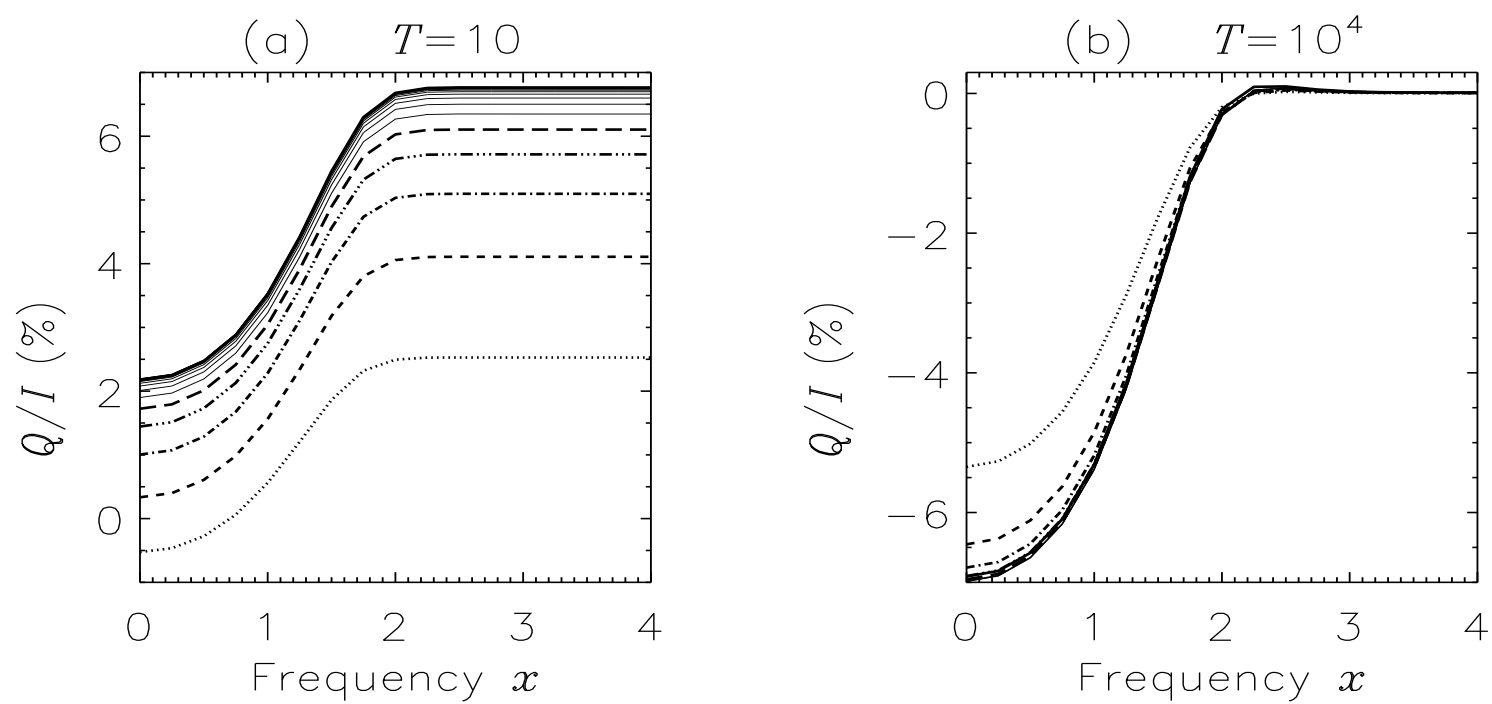

Fig. 5. Rayleigh scattering. Convergence history of the expansion method for the calculation of $Q / I$ shown for $\tau=0$ and $\mu=0.05$. Panels a) and b) correspond to $T=10$ and $T=10^{4}$ respectively. Different line types are: thick solid: exact; dotted: single scattering; dashed, dot-dashed, triple-dot dashed and long dashed: 2nd, 3rd, 4th, 5th and 6th iterations respectively. All the following iterations are plotted with thin solid lines.
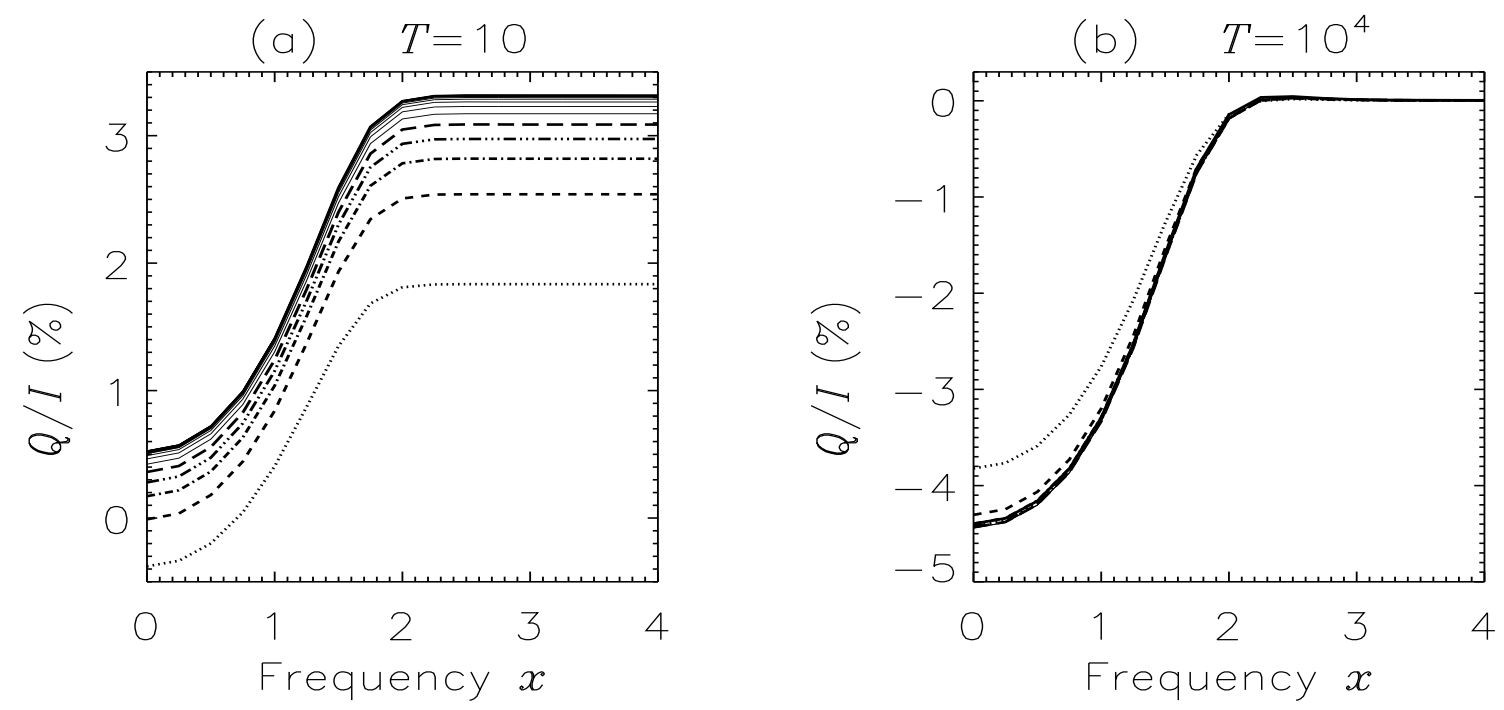

Fig. 6. Same as Fig. 5 but for a deterministic magnetic field with $\Gamma_{B}=1, \theta_{B}=30^{\circ}, \chi_{B}=45^{\circ}$.
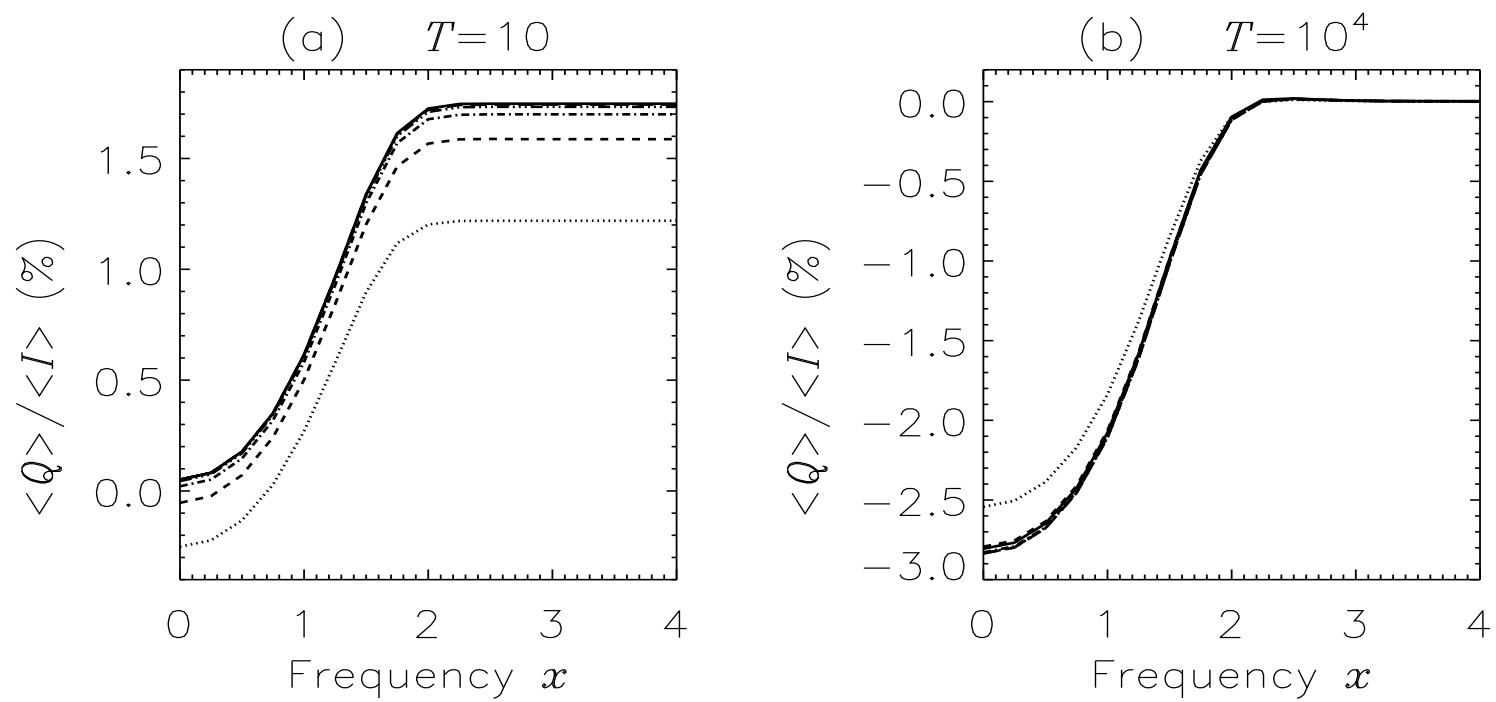

Fig. 7. Same as Figs. 5 and 6 but for a micro-turbulent magnetic with an isotropic angular distribution and single value field strength defined by $\Gamma_{B_{0}}=1$. 


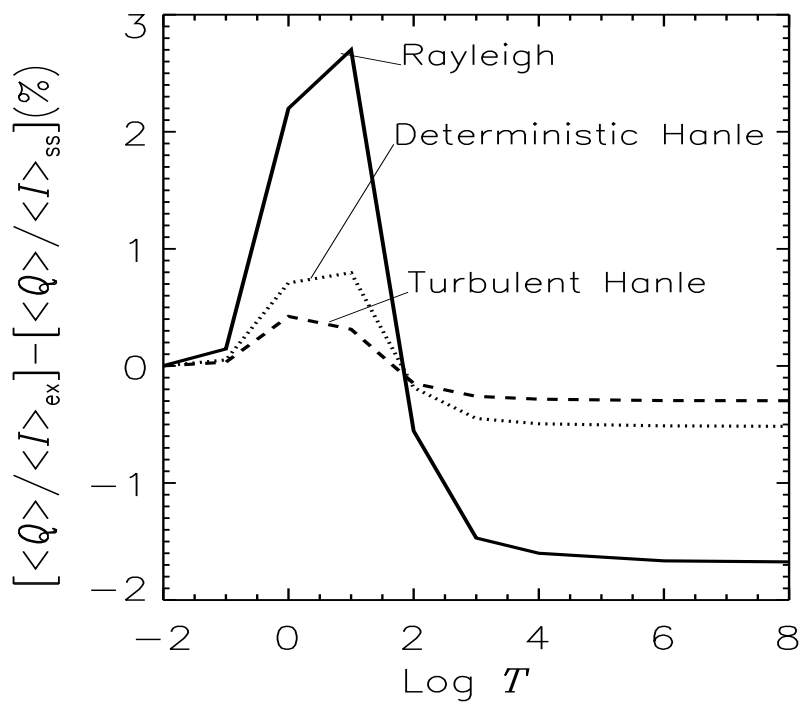

Fig. 8. Difference between single scattering (ss) and exact (ex) solution as a function of the optical thickness $T$ for $\tau=0, x=0$, and $\mu=$ 0.05. Rayleigh scattering (solid line), deterministic Hanle (dotted line), micro-turbulent Hanle (dashed line). Magnetic field parameters are the same as in Figs. 6 and 7.

the Hanle effect, the $N_{Q Q}^{2}$ and $\left\langle N_{Q Q}^{2}\right\rangle$ are significantly less than unity. Experiments with different angular distributions clearly show that a decrease in $\left\langle N_{00}^{2}\right\rangle$ induces a decrease in $e_{\mathrm{ss}}$.

Table 2 also shows clearly that the single scattering approximation is better for optically thin and optically thick lines than for lines with intermediate optical thicknesses. It also shows that this approximation is better for a micro-turbulent magnetic field than for a deterministic one or Rayleigh scattering. We examined the values of $N_{k}$ at different frequency points along the line profile and found that in the wings they are in general a bit higher than at line center.

Our last comment concerned the fact that the exact value of Stokes $Q$ is reached from below in the case of thin to moderately thick slabs and from above in the case of thick slabs (see Figs. 5 to 8$)$. The transition occurs around $T=10^{2}$ as shown in Fig. 8. This change of behavior is directly related to the sign of $C_{0}^{2}(\tau)$, determined by a competition between a limb-darkened outgoing radiation and a limb-brightened incoming one (see e.g. Trujillo Bueno 2001). For $T=10^{4}, C_{0}^{2}(\tau)$ is positive as long as $\tau$ is less than unity and then becomes negative ( $\tau$ is assumed to be in the range $0<\tau<T / 2)$. Since the sum inside the square bracket in Eq. (40) increases with $k$, the value of $\left[\tilde{S}_{0}^{2}\right]^{(k)}$ near the surface will also increase with the value of $k$. We can then deduce from Eq. (41) that $Q$ is negative and decreases (increases in absolute value) when $k$ increases.

For $T<1, C_{0}^{2}(\tau)$ is negative, so we have the opposite behavior. Apparently this behavior holds until $T$ becomes around $10^{2}$ (see Figs. 5 to 8 ) but we have no simple approximation for Stokes $Q$ or for $\left[\tilde{S}_{0}^{2}\right]^{(k)}(\tau)$, in this intermediate range of optical thicknesses.

What should be remembered is that the single scattering approximation can lead to very large errors for Rayleigh scattering, but may be sufficient for the micro-turbulent Hanle effect, especially when the line optical thickness is small or large enough.

\subsection{Magnetic field with a finite correlation length}

Assuming, as above, that $S_{0}^{0}$ is independent of the polarization and given by the solution of Eq. (33), the equation for $\tilde{\mathcal{S}}_{Q}^{2}(\tau \mid \boldsymbol{B})$ can be written as (see Eq. (A.1))

$$
\begin{aligned}
\tilde{\mathcal{S}}_{Q}^{2}(\tau \mid \boldsymbol{B})= & N_{Q 0}^{2}(\boldsymbol{B}) C_{0}^{2}(\tau) \\
& +\sum_{Q^{\prime}} N_{Q Q^{\prime}}^{2}(\boldsymbol{B})\left\{\int_{0}^{T} L_{Q^{\prime}}^{22}\left(\tau-\tau^{\prime} ; v\right) \tilde{\mathcal{S}}_{Q^{\prime}}^{2}\left(\tau^{\prime} \mid \boldsymbol{B}\right) \mathrm{d} \tau^{\prime}\right. \\
& +\int_{0}^{T}\left[K_{Q^{\prime}}^{22}\left(\tau-\tau^{\prime}\right)-L_{Q^{\prime}}^{22}\left(\tau-\tau^{\prime} ; v\right)\right] \\
& \left.\times \int P\left(\boldsymbol{B}^{\prime}\right) \tilde{\mathcal{S}}_{Q^{\prime}}^{2}\left(\tau^{\prime} \mid \boldsymbol{B}^{\prime}\right) \mathrm{d}^{3} \boldsymbol{B}^{\prime} \mathrm{d} \tau^{\prime}\right\}
\end{aligned}
$$

The iteration scheme defined in Eq. (37) can be carried out on this equation. If, at step $(k-1)$, one knows $\left[\tilde{\mathcal{S}}_{Q^{\prime}}^{2}\left(\tau^{\prime} \mid \boldsymbol{B}\right)\right]^{(k-1)}$, one also knows its average over the magnetic field PDF, which appears in the righthand side of Eq. (44). The iteration scheme for a finite value of $v$ will have the same convergence properties as the simpler case of Eq. (37).

\section{Dependence of the polarization on the magnetic field vector PDF}

This study is carried out for the micro-turbulent limit, because one can expect, from our previous results, that the dependence of the polarization on the shape of the magnetic field PDF will be essentially independent of the value of the correlation length.

In the micro-turbulent limit, the mean source vector satisfies Eq. (20). Here we are dealing with magnetic field distributions that are cylindrically symmetric about the vertical axis and a primary source term that is unpolarized. Hence, the matrix $\hat{N}(\boldsymbol{B})$ is diagonal and the only source vector components that are not zero are $\left\langle S_{0}^{0}\right\rangle$ and $\left\langle S_{0}^{2}\right\rangle$. For their calculation, carried out here with a standard PALI method, we only need $N_{00}^{0}$ and $\left\langle N_{00}^{2}\right\rangle$. The solution of Eq. (10), with $\left\langle S_{0}^{2}\right\rangle(\tau)$ as source term, yields $\left\langle I_{0}^{2}\right\rangle$. The mean value of Stokes $Q$ is then given by $\langle Q\rangle=-3\left(1-\mu^{2}\right) /(2 \sqrt{2})\left\langle I_{0}^{2}\right\rangle$.

The element $\left\langle N_{00}^{2}\right\rangle$ can be calculated explicitly for all the angular distributions given in Table 1 , when they are associated to the delta and Gaussian strength distributions. The expressions are given in Appendix B. In the other cases, $\left\langle N_{00}^{2}\right\rangle$ is calculated by numerical averaging with Gauss-Legendre quadratures. We have also considered a log-normal distribution, but it yields essentially the same results as the Gaussian distribution.

The calculations were performed for slabs with different optical thickness $T$ and we found that the main conclusions are essentially independent of the value of $T$. The results shown in this section correspond to a slab with parameters $\left(T, a, \epsilon^{\prime}, B_{v_{0}}\right)=$ $\left(10^{4}, 10^{-3}, 10^{-4}, 1\right)$.

Figure 9 is devoted to the isotropic distribution. Panel (a) shows that $\langle Q\rangle /\langle I\rangle$ increases (in absolute value) as we go from a Dirac distribution (single field strength value), to a Maxwell distribution, then a Gaussian distribution, and finally an exponential distribution, i.e. from case (i) to case (iv) (see Table 1). All these curves lie well above the Rayleigh scattering limit in which $Q / I(\tau=0, x=0, \mu=0.05)=-0.07$. The variation of $\langle Q\rangle /\langle I\rangle$ is due to the fact that the value of $\left\langle N_{00}^{2}\right\rangle$ increases as we go from case (i) to case (iv), because the probability of having weak magnetic fields increases. The maximum value of $\left\langle N_{00}^{2}\right\rangle$ is reached for Rayleigh scattering.

In Fig. 9b we show the center-to-limb variation of $(\langle Q\rangle /\langle I\rangle) /\left(1-\mu^{2}\right)$. A striking feature is that the variation with $\mu$ is almost insensitive to the field strength distribution. We have even 

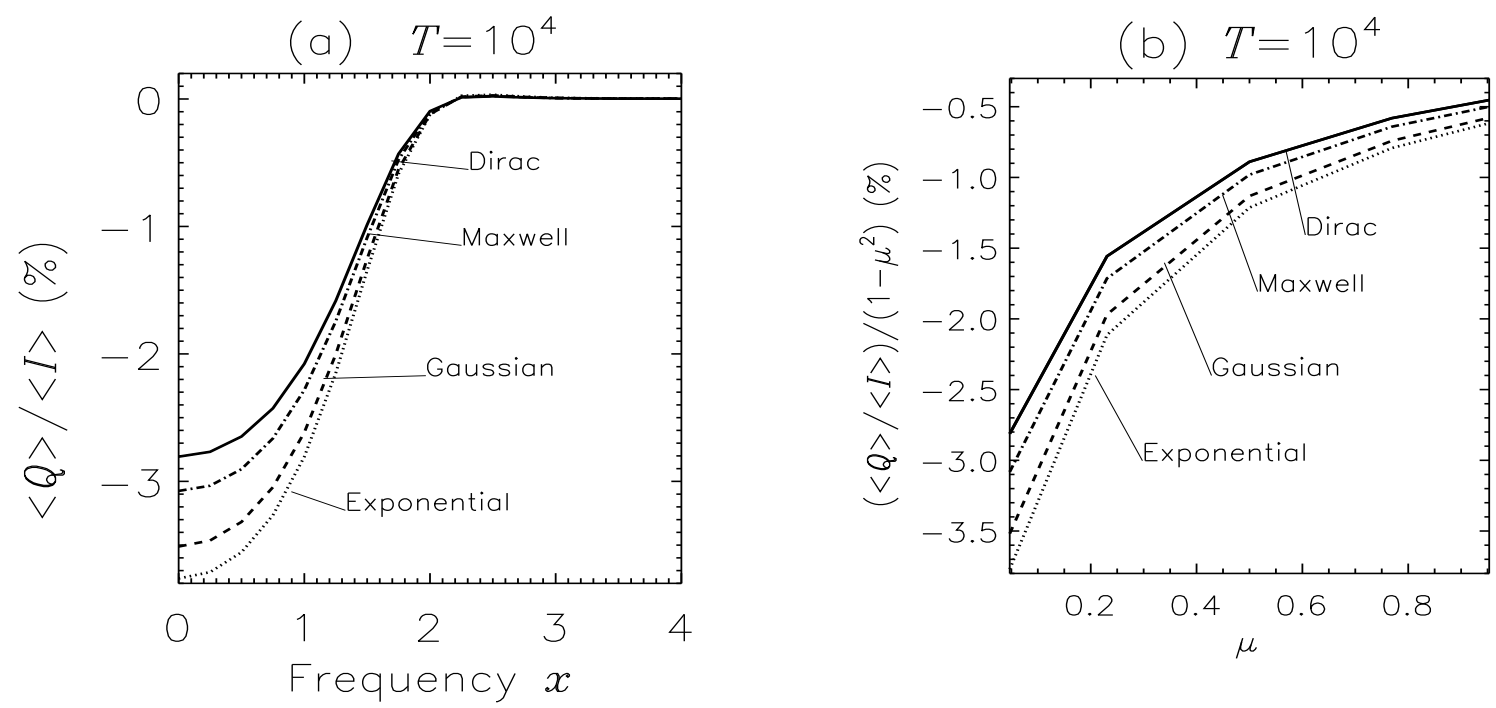

Fig. 9. Panel a): profile of $\langle Q\rangle /\langle I\rangle$, for $\tau=0$ and $\mu=0.05$, calculated with the isotropic distribution combined with different $P_{\mathrm{S}}(B)$. Panel b): variation with $\mu$, for $\tau=0, x=0$, and the same PDFs. Solid line: $P_{\mathrm{D}}\left(B / B_{0}\right)$; dot-dashed line: $P_{\mathrm{M}}\left(B / B_{0}\right)$; dashed line: $P_{\mathrm{G}}\left(B / B_{0}\right)$; dotted line: $P_{\mathrm{E}}\left(B / B_{0}\right)$. Model parameters of the slab $\left(T, a, \epsilon^{\prime}, B_{v_{0}}\right)=\left(10^{4}, 10^{-3}, 10^{-4}, 1\right)$.
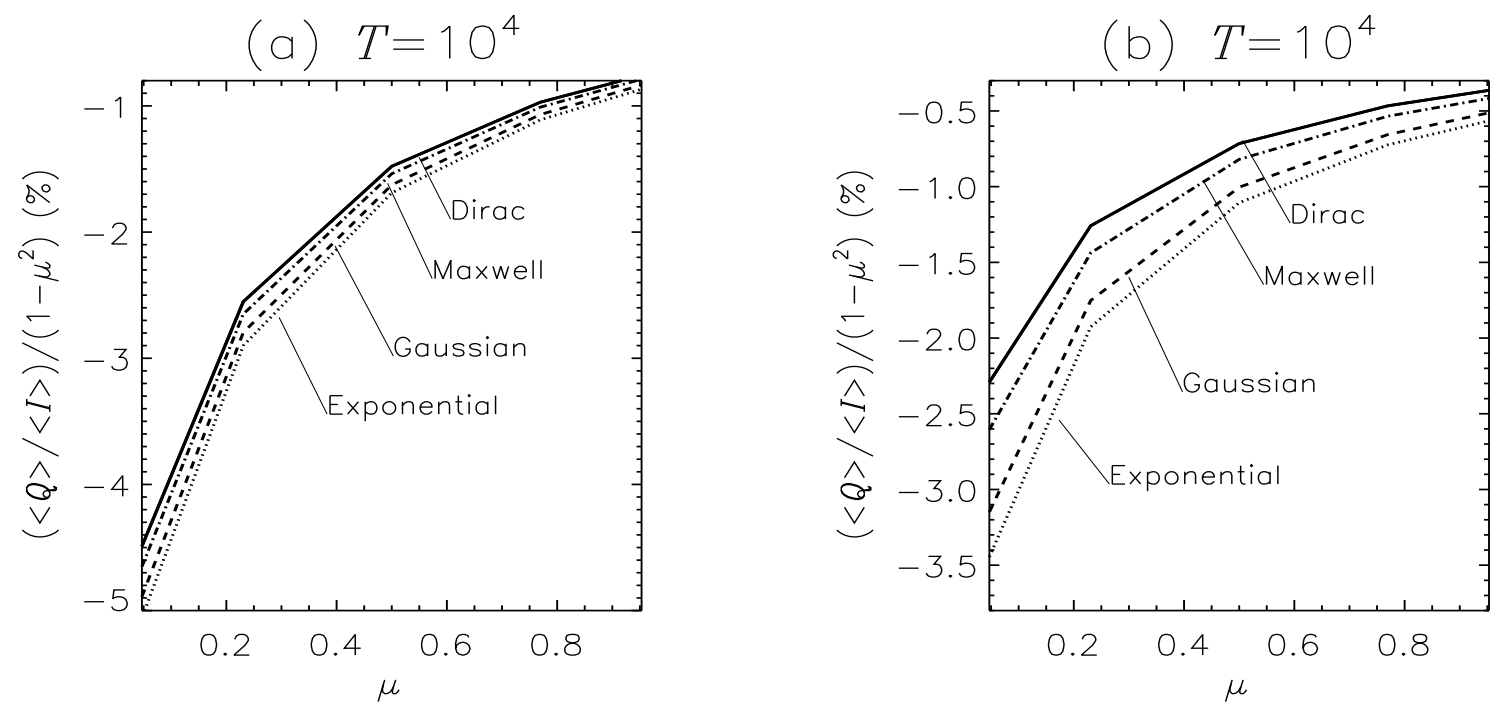

Fig. 10. Panel a): variation of the ratio $(\langle Q\rangle /\langle I\rangle) /\left(1-\mu^{2}\right)$ with respect to $\mu$, at $\tau=0, x=0$, for the cosine angular power law, with $p=5$. Panel b): same results but for the sine power law with $p=50$. Each of the angular distributions is combined with different strength distributions. Solid line: $P_{\mathrm{D}}\left(B / B_{0}\right)$; dot-dashed line: $P_{\mathrm{M}}\left(B / B_{0}\right)$; dashed line: $P_{\mathrm{G}}\left(B / B_{0}\right)$; dotted line: $P_{\mathrm{E}}\left(B / B_{0}\right)$. Model parameters of the slab $\left(T, a, \epsilon^{\prime}, B_{v_{0}}\right)=$ $\left(10^{4}, 10^{-3}, 10^{-4}, 1\right)$.

found that the full line curve, corresponding to a Dirac PDF with $B=B_{0}$, coincides exactly with the center-to-limb variation given by an exponential distribution with a mean value $\langle B\rangle=2 B_{0}$. This result fully agrees with the calculations of Trujillo Bueno et al. (2004), showing that observed center-to-limb variations can be fitted by an isotropic field with a strength of $60 \mathrm{G}$, or by an exponential distribution with a mean value of $130 \mathrm{G}$.

Somewhat more insight into the behavior of $\langle Q\rangle$ can be obtained by considering $\left\langle S_{0}^{2}\right\rangle(\tau)$. The dependence on optical depth is controlled by the propagation kernel $K_{0}^{22}(\tau)$ (see Sect. 7). As a result, changing the shape of $P_{\mathrm{S}}(B)$ will have a very small effect on the $\mu$-dependence of $\langle Q\rangle$ (see Eq. (41)). In contrast, a change in the shape of $P_{\mathrm{S}}(B)$ will modify the value of $\left\langle N_{00}^{2}\right\rangle$, hence the degree of polarization.

In Fig. 10, devoted to the cosine and sine angular power laws, we see that the ratio $(\langle Q\rangle /\langle I\rangle) /\left(1-\mu^{2}\right)$ also increases from case (i) to case (iv). The dependence on the shape of $P_{\mathrm{S}}(B)$ is quite strong for the sine power law with $p=50$ (even a bit more than with the isotropic distribution), but very small for the cosine power law with $p=5$. This stems from the reduction of the Hanle effect when the field becomes strongly peaked in the vertical direction.

In Fig. 11, we show the ratio $(\langle Q\rangle /\langle I\rangle) /\left(1-\mu^{2}\right)$ for magnetic fields with different angular distributions, the field strength being kept equal to a single value $B_{0}$. In Fig. $11 \mathrm{a}$, we see that the choice of the angular distribution has a strong effect on the amplitude of this ratio, but not on its center-to-limb variation, for the reason given above. Figure $11 \mathrm{~b}$ shows the variation in this ratio with the Hanle efficiency parameter $\Gamma_{B_{0}}$ for $\tau=0, x=0$, and $\mu=0.05$. We observe the standard Hanle saturation for large field strengths. An isotropic distribution, and a sine power law with a fairly horizontally peaked distribution, yield similar polarizations, as already been pointed out in Stenflo (1982). There are, however, observable differences around $\Gamma_{B_{0}}=1$. The 

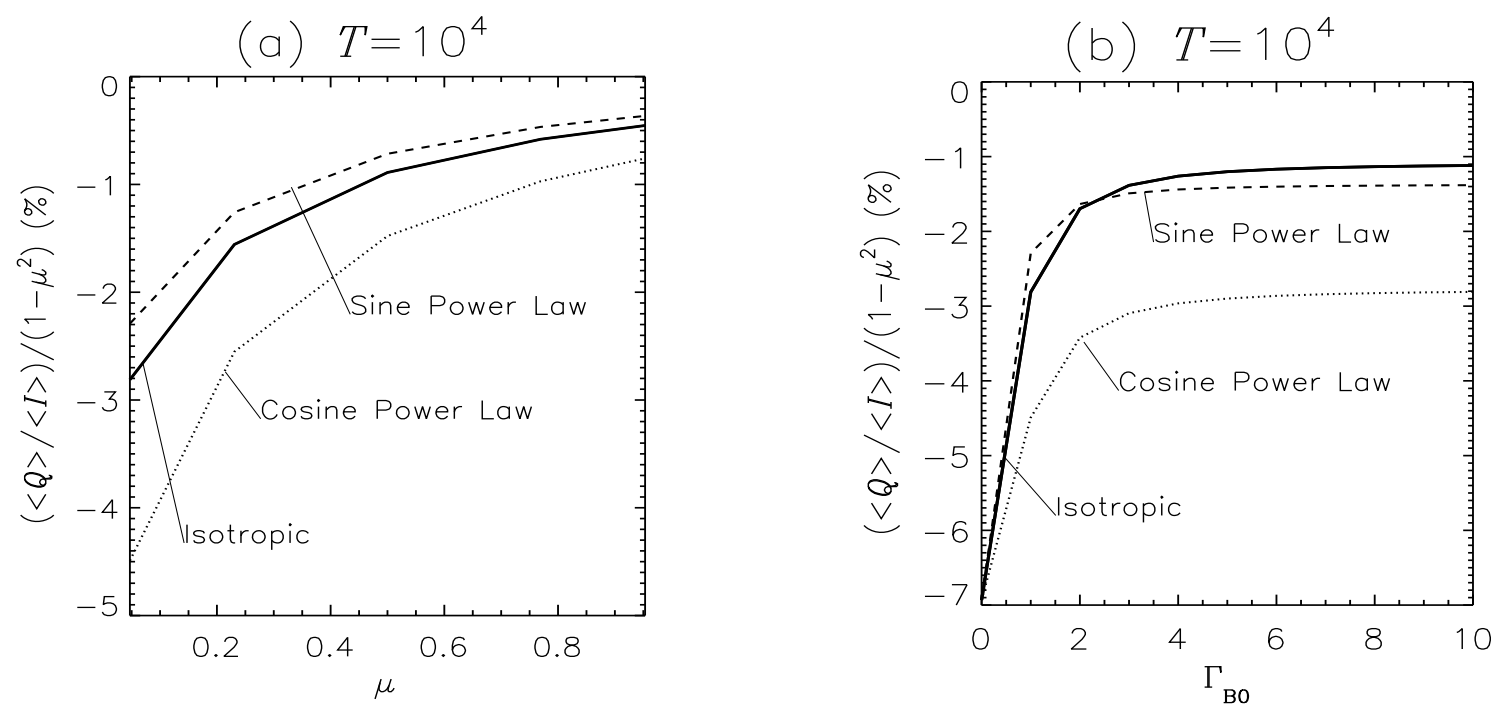

Fig. 11. Panel a): variation of the ratio $(\langle Q\rangle /\langle I\rangle) /\left(1-\mu^{2}\right)$ with respect to $\mu$, at $\tau=0, x=0$, for the isotropic distribution, cosine power law with $p=5$, and sine power law with $p=50$; the magnetic field strength has a single value corresponding to $\Gamma_{B_{0}}=1$. Panel $\left.\mathbf{b}\right)$ : dependence on $\Gamma_{B_{0}}$ for $\tau=0, x=0, \mu=0.05$. Model parameters of the slab $\left(T, a, \epsilon^{\prime}, B_{v_{0}}\right)=\left(10^{4}, 10^{-3}, 10^{-4}, 1\right)$.

polarization is higher for the cosine power law because the distribution is strongly peaked in the vertical direction.

These numerical experiments with different magnetic field PDFs indicate a clear sensitivity of the polarization to the magnetic field strength and angular distributions. Hence, any information on mean magnetic field strengths, extracted from Hanle depolarization measurements, may depend critically on the choice of the magnetic field PDF that has been made a priori for the analysis of the observations.

\section{Concluding remarks}

In this paper, we have studied the Hanle effect due to a random magnetic field with a finite correlation length, in order to assess limitations to the usual micro-turbulent approximation. The modeling of the magnetic field by a Markovian random process, piecewise constant, characterized by a correlation length and a magnetic field vector probability density function (PDF), enabled us to construct a radiative transfer equation for a mean radiation field, which still depends on the random values of the magnetic field (Sect. 3). A simple averaging of the solution of this equation over the PDF yields the mean Stokes parameters. The transfer equation is solved numerically by a PALI method, generalized to the problem at hand (Sect. 4).

We find that optically thin lines (lines with optical thickness $T \ll 1)$, and very optically thick ones $(T \gg 100)$ can be treated with the micro-turbulent approximation. For these lines, the polarization is created locally by a small number of scattering events. For optically thick lines they are located near the surface. To evaluate this number of events, the polarization has been calculated by a method of successive iterations leading to a series expansion in the mean number of scattering events (Sect. 7). For optically thin and thick lines, this number is around 5; for lines with intermediate optical thicknesses $(T \simeq 10-100)$, it is significantly more (10-15) and these lines show some sensitivity to the magnetic field correlation length (see Fig. 4).

We also find that for a random magnetic field, the single scattering approximation can be safely used to evaluate the Hanle depolarization. For a deterministic magnetic field, it may also provide a reasonable approximation. In contrast, for the Rayleigh scattering, it may lead to large errors, except for optically thin lines (see Fig. 8).

Numerical experiments carried out in the micro-turbulent limit, with different types of magnetic field PDF, indicate that the polarization is quite sensitive to the shape of the PDF (Sect. 8). However, our results suggest that it may not be easy to retrieve a quiet Sun magnetic field PDF from the Hanle effect depolarization measurements, since the same degree of linear polarization can be created by PDFs that have rather different shapes. The center-to-limb variation of the linear polarization also depends very little on the PDF shape. Several laws for the solar magnetic field PDF have been proposed in recent years. They have been deduced from Zeeman effect measurements and may contain some uncertainty in the weak field domain involved in the Hanle effect. Numerical simulations such as those carried out in Schüssler \& Vögler (2008) may clarify the situation.

In this paper, we have complete frequency redistribution at each scattering. This assumption is certainly not valid to analyze the Hanle depolarization of strong resonance lines showing significant partial redistribution effects. An example is the Ba II D2 line considered in Faurobert et al. (2009) to evaluate the turbulent magnetic field in the low chromosphere. However, our conclusions concerning the applicability of the micro-turbulent approximation remain most probably valid, since the polarization is still created in a small region close to the surface. The transfer equations given here and their method of solution can be easily generalized to handle partial frequency redistribution and to verify this prediction, but this generalization will be accompanied by a significant increase in computing time.

Acknowledgements. The authors have greatly benefited from discussions with V. Bommier and J. O. Stenflo. L.S.A. is grateful to the Laboratoire Cassiopée (Université de Nice, OCA, CNRS) for financial support and hospitality during a stay in Nice where part of this work was completed.

\section{Appendix A: Integral equations for the components of the source vector}

In Eqs. (17) to (19) of the text, we give the integral equation for the source vector $\mathcal{S}(\tau \mid \boldsymbol{B})$. The corresponding system of integral 
equations for its $K Q$ components $\mathcal{S}_{Q}^{K}(\tau \mid \boldsymbol{B})$ may be written as

$$
\begin{aligned}
\mathcal{S}_{Q}^{K}(\tau \mid \boldsymbol{B})= & \mathcal{G}_{Q}^{K}(\tau) \\
& +\sum_{K^{\prime} Q^{\prime}} N_{Q Q^{\prime}}^{K}(\boldsymbol{B}) \int_{0}^{T}\left\{L_{Q^{\prime}}^{K K^{\prime}}\left(\tau-\tau^{\prime} ; v\right) \mathcal{S}_{Q^{\prime}}^{K^{\prime}}\left(\tau^{\prime} \mid \boldsymbol{B}\right)\right. \\
& +\left[L_{Q^{\prime}}^{K K^{\prime}}\left(\tau-\tau^{\prime} ; 0\right)-L_{Q^{\prime}}^{K K^{\prime}}\left(\tau-\tau^{\prime} ; v\right)\right] \\
& \left.\times \int P\left(\boldsymbol{B}^{\prime}\right) \mathcal{S}_{Q^{\prime}}^{K^{\prime}}\left(\tau^{\prime} \mid \boldsymbol{B}^{\prime}\right) d^{3} \boldsymbol{B}^{\prime}\right\} \mathrm{d} \tau^{\prime},
\end{aligned}
$$

with

$L_{Q^{\prime}}^{K K^{\prime}}(\tau ; v)=\int_{-\infty}^{+\infty} \int_{0}^{1} \frac{1}{2 \mu} \Psi_{Q^{\prime}}^{K K^{\prime}}(\mu) \mathrm{e}^{-\frac{|\tau|}{\mu}(\varphi(x)+v)} \varphi^{2}(x) \mathrm{d} \mu \mathrm{d} x$.

The $\Psi_{Q^{\prime}}^{K K^{\prime}}(\mu)$ are real, even functions of $\mu$ and satisfy $\Psi_{Q^{\prime}}^{K K^{\prime}}(\mu)=$ $\Psi_{-Q^{\prime}}^{K K^{\prime}}(\mu)$ (LL04, Appendix A20). For a non polarized primary source term,

$\mathcal{G}_{Q}^{K}(\tau)=\delta_{K 0} \delta_{Q 0} G(\tau)$,

with $G(\tau)$ proportional to the Planck function. For a two-level atom with unpolarized ground level, the elements $N_{Q Q^{\prime}}^{K}(\boldsymbol{B})$ of the matrix $\hat{N}(\boldsymbol{B})$ can be written as

$N_{Q Q^{\prime}}^{K}\left(B, \theta_{B}, \chi_{B}\right)=\mathrm{e}^{\mathrm{i}\left(Q^{\prime}-Q\right) \chi_{B}} M_{Q Q^{\prime}}^{K}\left(B, \theta_{B}\right)$,

with

$M_{Q Q^{\prime}}^{K}\left(B, \theta_{B}\right)=\sum_{Q^{\prime \prime}} d_{Q Q^{\prime \prime}}^{K}\left(\theta_{B}\right) d_{Q^{\prime \prime} Q^{\prime}}^{K}\left(-\theta_{B}\right) X_{K Q^{\prime \prime}}(B)$.

We recall that $d_{Q Q^{\prime \prime}}^{K}\left(\theta_{B}\right)$ are the reduced rotation matrices, with $\theta_{B}$ and $\chi_{B}$ the polar angles (inclination and azimuth) of the magnetic field direction in an atmospheric reference frame with the $z$-axis along the normal to the surface (see Fig. 1 in the text). Explicit expressions for $X_{K Q^{\prime \prime}}(B)$ can be found in LL04 (Chaps. 5, 10, 14; see also HF07). They can be written as

$X_{K Q^{\prime \prime}}(B)=\frac{W_{K}\left(J_{1}, J_{\mathrm{u}}\right)}{1+\epsilon+\delta_{\mathrm{u}}^{(K)}}\left[\frac{1}{1+Q^{\prime \prime} \Gamma_{B}^{\prime}}\right]$.

Here $\Gamma_{B}^{\prime}=\Gamma_{B} /\left(1+\epsilon+\delta_{\mathrm{u}}^{(K)}\right)$ with $\epsilon=C_{\mathrm{u}, 1} / A_{\mathrm{u}, 1}$ and $\delta_{\mathrm{u}}^{(K)}=D^{(K)} / A_{\mathrm{u}, 1}$ (Bommier 1997; LL04 p. 520 and 532). We recall that $C_{\mathrm{u}, 1}$ and $A_{\mathrm{u}, 1}$ are the inelastic collisional and radiative de-excitation rates. The parameter $D^{(K)}$, with $D^{(0)}=0$, is the effective number of depolarizing collisions, for the statistical tensor of rank $K$, taking place during the lifetime of the excited level. The magnetic field strength $B$ enters through the efficiency factor $\Gamma_{B}=2 \pi v_{\mathrm{L}} g_{\mathrm{u}} / A_{\mathrm{u}, 1}=\left(e_{0} / 2 m c\right)\left(g_{\mathrm{u}} / A_{\mathrm{u}, 1}\right) B$ where $v_{\mathrm{L}}$ is the Larmor frequency, $e_{0}$ and $m$ the charge and mass of the electron, $c$ the speed of light and $g_{\mathrm{u}}$ the Lande factor of the upper level. Finally, $W_{K}\left(J_{1}, J_{\mathrm{u}}\right)$ are atomic depolarization parameters that can be found in LL04 (Table 10.1, p. 515). For a normal Zeeman triplet $\left(J_{1}=0, J_{\mathrm{u}}=1\right), W_{K}=1$ for all values of $K$. Explicit expressions of the $N_{Q Q^{\prime}}^{K}$ can be found in HF07 (see also FaurobertScholl 1991; Nagendra et al. 1998).

Equation (A.4) shows that the $\chi_{B}$ dependence of the $\mathcal{S}_{Q}^{K}$ appears as a phase factor. This suggests introducing a new function $S_{Q}^{K}\left(\tau \mid B, \theta_{B}\right)$ defined by the relation

$\mathcal{S}_{Q}^{K}\left(\tau \mid B, \theta_{B}, \chi_{B}\right)=\mathrm{e}^{-\mathrm{i} Q \chi_{B}} S_{Q}^{K}\left(\tau \mid B, \theta_{B}\right)$.
The integral equation for this new function is

$$
\begin{aligned}
S_{Q}^{K}(\tau \mid B, & \left.\theta_{B}\right)=\delta_{K 0} \delta_{Q 0} G(\tau) \\
& +\sum_{K^{\prime} Q^{\prime}} M_{Q Q^{\prime}}^{K}\left(B, \theta_{B}\right) \int_{0}^{T}\left\{L_{Q^{\prime}}^{K K^{\prime}}\left(\tau-\tau^{\prime} ; v\right) S_{Q^{\prime}}^{K^{\prime}}\left(\tau^{\prime} \mid B, \theta_{B}\right)\right. \\
& +\left[L_{Q^{\prime}}^{K K^{\prime}}\left(\tau-\tau^{\prime} ; 0\right)-L_{Q^{\prime}}^{K K^{\prime}}\left(\tau-\tau^{\prime} ; v\right)\right] \\
& \times \oint \mathrm{e}^{-\mathrm{i} Q^{\prime}\left(\chi_{B}^{\prime}-\chi_{B}\right)} P\left(B^{\prime}, \theta_{B}^{\prime}, \chi_{B}^{\prime}\right) S_{Q^{\prime}}^{K^{\prime}}\left(\tau^{\prime} \mid B^{\prime}, \theta_{B}^{\prime}\right) \\
& \left.\times B^{\prime 2} \sin \theta_{B}^{\prime} d \theta_{B}^{\prime} \mathrm{d} B^{\prime} \frac{\mathrm{d} \chi_{B}^{\prime}}{4 \pi}\right\} \mathrm{d} \tau^{\prime} .
\end{aligned}
$$

This equation becomes simpler if the magnetic field PDF is cylindrically symmetrical with respect to the $z$-axis, i.e. of the form

$P(\boldsymbol{B}) \mathrm{d}^{3} \boldsymbol{B}=h\left(B, \theta_{B}\right) B^{2} \sin \theta_{B} \mathrm{~d} \theta_{B} \mathrm{~d} B \frac{\mathrm{d} \chi_{B}}{4 \pi}$.

We can integrate over $\chi_{B}^{\prime}$ the last term in Eq. (A.8). It will be zero, unless $Q^{\prime}=0$. Equation (A.8) reduces thus to

$$
\begin{aligned}
S_{Q}^{K}(\tau \mid B, & \left.\theta_{B}\right)=\delta_{K 0} \delta_{Q 0} G(\tau) \\
& +\sum_{K^{\prime} Q^{\prime}} M_{Q Q^{\prime}}^{K}\left(B, \theta_{B}\right) \int_{0}^{T}\left\{L_{Q^{\prime}}^{K K^{\prime}}\left(\tau-\tau^{\prime} ; v\right) S_{Q^{\prime}}^{K^{\prime}}\left(\tau^{\prime} \mid B, \theta_{B}\right)\right. \\
& +\delta_{Q^{\prime} 0}\left[\left[L_{Q^{\prime}}^{K K^{\prime}}\left(\tau-\tau^{\prime} ; 0\right)-L_{Q^{\prime}}^{K K^{\prime}}\left(\tau-\tau^{\prime} ; v\right)\right]\right. \\
& \times \int_{B^{\prime}} \frac{1}{2} \int_{0}^{\pi} h\left(B^{\prime}, \theta_{B}^{\prime}\right) S_{Q^{\prime}}^{K^{\prime}}\left(\tau^{\prime} \mid B^{\prime}, \theta_{B}^{\prime}\right) \\
& \left.\left.\times B^{\prime 2} \sin \theta_{B}^{\prime} \mathrm{d} \theta_{B}^{\prime} \mathrm{d} B^{\prime}\right]\right\} \mathrm{d} \tau^{\prime}
\end{aligned}
$$

We note here that the term involving the mean value of $S_{Q^{\prime}}^{K^{\prime}}$ is zero when $Q^{\prime} \neq 0$.

Once the $S_{Q}^{K}\left(\tau \mid B, \theta_{B}\right)$ have been calculated, they have to be multiplied by $\mathrm{e}^{-\mathrm{i} Q \chi_{B}}$ (see Eq. (A.7)) and then averaged over the magnetic field PDF. Since $P(\boldsymbol{B})$ has been assumed to have a cylindrical symmetry, the averaging process will give zero, except for the components with $Q=0$, so the mean source vector $\langle\mathcal{S}\rangle(\tau)$ and mean Stokes vector $\langle\mathcal{I}\rangle(\tau, x, \mu)$ only have two nonzero components corresponding to $K=0,2$ and $Q=0$. The magnetic field PDF does not break the cylindrically symmetry of the atmosphere. We stress that there is no way to avoid the calculations of the $S_{Q}^{K}\left(\tau \mid B, \theta_{B}\right)$ components with $Q \neq 0$. The reason is that the integral equation for the conditional mean source vector holds for both the micro and macro-turbulent limits.

\section{Appendix B: Exact expressions of the mean coefficient $\left\langle N_{00}^{2}\right\rangle$}

The coefficient $N_{00}^{2}$ is defined in the Appendix A. Exact expressions for the mean values $\left\langle N_{00}^{2}\right\rangle$ can obtained with the PDFs given in Table 1, when the magnetic field strength has a Dirac or Gaussian distribution. Because of the cylindrical symmetry of the PDFs, $\left\langle N_{00}^{2}\right\rangle=\left\langle M_{00}^{2}\right\rangle$. The expressions given below correspond to $W_{K}=1$ and $\delta_{u}^{(K)}=0$.

When the field strength has a Dirac distribution, the coefficents $\left\langle N_{00}^{2}\right\rangle$ have the form

$$
\left\langle N_{00}^{2}\right\rangle_{\mathrm{D}}=\frac{1}{1+\epsilon}\left[1-C_{1}\left(\frac{\Gamma_{B}^{\prime 2}}{1+\Gamma_{B}^{\prime 2}}\right)-C_{2}\left(\frac{4 \Gamma_{B}^{\prime 2}}{1+4 \Gamma_{B}^{\prime 2}}\right)\right] .
$$


Table B.1. Coefficients $C_{1}$ and $C_{2}$ for the calculation of $\left\langle N_{00}^{2}\right\rangle$.

\begin{tabular}{cccc}
\hline \hline & Isotropic & Cosine power law & Sine power law \\
\hline$C_{1}$ & $\frac{2}{5}$ & $\frac{6(p+1)}{(p+3)(p+5)}$ & $\frac{3(p+2)}{(p+3)(p+5)}$ \\
$C_{2}$ & $\frac{2}{5}$ & $\frac{6}{(p+3)(p+5)}$ & $\frac{3(p+2)(p+4)}{4(p+3)(p+5)}$ \\
\hline
\end{tabular}

The coefficients $C_{1}$ and $C_{2}$ only depend on the angular distribution. The coefficient $\Gamma_{B}^{\prime}$ is defined in Appendix A. The index $B$ stands for $B_{0}$.

When the field strength has a Gaussian distribution,

$\left\langle N_{00}^{2}\right\rangle_{\mathrm{G}}=\frac{1}{1+\epsilon}\left[1-C_{1} K_{1}-C_{2} K_{2}\right]$.

The coefficients $K_{m}, m=1,2$, may be written as

$K_{m}=1-\left[\frac{\sqrt{\pi}}{m \gamma_{\sigma}} \exp \left(\frac{1}{\left(m \gamma_{\sigma}\right)^{2}}\right) \operatorname{erfc}\left(\frac{1}{m \gamma_{\sigma}}\right)\right]$,

with

$\gamma_{\sigma}=\sqrt{\pi} \Gamma_{B}^{\prime}$.

One can check that the coefficients $K_{1}$ and $K_{2}$ go to zero when the magnetic field goes to zero.

We give in Table B. 1 the coefficients $C_{1}$ and $C_{2}$ for the isotropic, cosine, and sine power laws defined in Table 1, Col. 2 , of the text. Some of these results can be found in Stenflo (1982, 1994, Eq. (10.54)).

For $p=0, C_{1}$ and $C_{2}$ go to $2 / 5$ and we recover the isotropic angular distribution. For the cosine power law, when $p$ goes to infinity, $C_{1}$ and $C_{2}$ go to zero and we recover the Rayleigh scattering. For the sine power law, when $p$ goes to infinity, $C_{1}$ goes to zero and $C_{2}$ to 0.75 .

\section{References}

Auer, L. H., \& Paletou, F. 1994, A\&A, 285, 675

Auvergne, M., Frisch, H., Frisch, U., Froeschlé, Ch., \& Pouquet, A. 1973, A\&A, 29, 93

Bommier, V. 1997, A\&A, 328, 726

Bommier, V., Derouich, M., Landi Degl'Innocenti, E., Molodij, G., \& SahalBréchot, S. 2005, A\&A, 432, 295
Brissaud, A., \& Frisch, U. 1971, JQSRT, 11, 1767

Brissaud, A., \& Frisch, U. 1974, J. Math. Phys., 15, 524

Dominguez Cerdeǹa, I., Sánchez Almeida, J., \& Kneer, F. 2006, ApJ, 646, 1421

Carroll, T. A., \& Staude, J. 2005, Astron. Nachr., 326, 296

Faurobert-Scholl, M. 1991, A\&A, 246, 469

Faurobert-Scholl, M. 1993, A\&A, 268, 765

Faurobert-Scholl, M. 1996, Sol. Phys. 164, 79

Faurobert, M., Arnaud, J., Vigneau, J., \& Frisch, H. 2001, A\&A, 378, 627

Faurobert, M., Derouich, M., Bommier, V., \& Arnaud, J. 2009, A\&A, 493, 201

Fluri D. M., Nagendra K. N., \& Frisch H. 2003, A\&A, 400, 303

Frisch, H. 2006, A\&A, 446, 403 (HF06)

Frisch, H. 2007, A\&A, 476, 665 (HF07)

Frisch, H., \& Frisch, U. 1975, in Colloque International du CNRS, ed. R. Cayrel,

\& M. Steinberg (Editions du CNRS), 250, 113

Frisch, H., \& Frisch, U. 1976, MNRAS, 175, 157

Iyanaga, S., \& Kawada, Y. 1970, Encyclopedic Dictionary of Mathematics (Cambridge, Massachusetts: The MIT Press)

Kunasz, P. B., \& Auer, L. H. 1988, JQSRT, 39, 67

Landi Degl'Innocenti, E. 1984, Sol. Phys., 91, 1

Landi Degl'Innocenti, E., \& Bommier, V. 1994, A\&A, 284, 865

Landi Degl'Innocenti, E., \& Landolfi, M. 2004, Polarization in Spectral Lines (Kluwer Academic Publishers) (LL04)

Landi Degl'Innocenti, E., Bommier, V., \& Sahal-Bréchot, S. 1990, A\&A, 235, 459

Léger, L., Chevallier, L., \& Paletou, F. 2007, A\&A, 470, 1

Lites, B., Kubo, M., Socas-Navarro, H., et al. 2008, ApJ, 672, 1237

Manso Sainz, R., \& Trujillo Bueno, J. 1999, ASSL Ser., 243, Solar Polarization, ed. K. N. Nagendra, \& J. O. Stenflo, 143

Manso Sainz, R., \& Trujillo Bueno, J. 2003, ASP Conf. Ser. 307, ed. J. Trujillo Bueno, \& J. Sánchez Almeida, 251

Nagendra, K. N., Frisch, H., \& Faurobert-Scholl, M. 1998, A\&A, 332, 610

Nagendra, K. N., Paletou, F., Frisch, H., \& Faurobert-Scholl, M. 1999, Solar Polarization II, ed. K. N. Nagendra, \& J. O. Stenflo (Boston: Kluwer Academic Publishers), 127

Nagendra, K. N., \& Sampoorna, M. 2009, in Solar Polarization 5, ed. S. V. Berdyugina, K. N. Nagendra, \& R. Ramelli, ASP Conf. Ser., 405, 261

Papoulis, A. 1965, Probability, Random Variables, and Stochastic Processes (New York: Mac Graw Hill)

Sampoorna, M., Nagendra, K. N., \& Frisch, H. 2008a, JQSRT, 109, 2349

Sampoorna, M., Nagendra, K. N., Frisch, H., \& Stenflo, J. O. 2008b, A\&A, 485, 275

Sánchez Almeida, J. 2007, ApJ, 657, 1150

Schüssler, M., \& Vögler, A. 2008, A\&A, 481, L5

Stenflo, J. O. 1982, Sol. Phys., 80, 209

Stenflo, J. O. 1987, Sol. Phys., 114, 1

Stenflo, J. O. 1994, Solar Magnetic Fields (Dordrecht: Kluwer)

Stenflo, J. O. 2009, in Astrophys. Space Science Proceedings, Magnetic coupling between the Interior and Atmospheres of the Sun, ed. S. S. Hasan, \& R. J. Rutten, in press

Stoer, J., \& Bulirsch, R. 1983, Introduction to Numerical Analysis (SpringerVerlag)

Trujillo Bueno, J. 2001, in Advanced Solar Polarimetry Theory, Observation, and Instrumentation, ed. M. Sigwarth, ASP Conf. Ser., 236, 161

Trujillo Bueno, J., \& Fabiani Bendicho, P. 1995, ApJ, 455, 646

Trujillo Bueno, J., Shchukina, N., \& Asensio Ramos, A. 2004, Nature, 430, 326 